\title{
A New Mass Spectrograph
}

\author{
Takekiyo Matsuo \\ Institute of Physics, College of General Education, Osaka University, Toyonaka, Osaka, Japan
}

\author{
Morio Ishihara \\ Analytical Instruments Division, JEOL Ltd., Akishima, Tokyo, Japan
}

The optical designs of two new types of mass spectrographs were studied. The first is a system that possesses a specially shaped magnet output boundary to satisfy the doublefocusing condition for a wide mass range. The focal plane is usually curved. The second system is one in which a parallel ion beam is generated before the magnet, forming a straight double-focusing line. By introducing a quadrupole lens doublet such that the ion beam may be deflected in the same direction through the electric and magnetic fields, the overall image magnification can be arbitrarily controlled and stigmatic focusing achieved for the median ray. (I Am Soc Mass Spectrom 1993, 4, 372-386)

$\mathrm{T}$ The areas of application in mass spectrometry have been seen over recent years to be evolving into five categories: (1) high resolution, (2) high mass range, (3) high sensitivity, (4) coupling of gas chromatographic/liquid chromatographic systems with mass spectrometry, and (5) tandem mass spectrometry including collision-activated dissociation/ mass spectrometry. In this report we focus on the third category: "high sensitivity." There have been considerable recent developments in liquid chromatographic interfaces and array detectors. Now, the order of picomoles or femtomoles of the sample may be ionized directly from the liquid phase, and the use of nonscanning detectors (arrays) has increased detection efficiency at least by two orders of magnitude. Thus, it is important to design a new mass spectrograph to increase the sensitivity and to use these new ion sources and detectors more efficiently. "A mass spectrograph" is a sector-type mass analyzer without electric or magnetic scanning.

Because constant energy beams are analyzed in normal mass spectrometry, we discuss only the system where an electrostatic analyzer (ESA) comes first and a magnet must follow it (forward arrangement). To understand mass spectrometers/graphs systematically, it may be helpful to introduce two types of classifications:

1. The direction of deflection of an ESA and a magnet that are the same (normal) or inverse. This relates

Address reprint requests to Takekiyo Matsuo, Institute of Physics, College of General Education, Osaka University, Toyonaka, Osaka 560 , Japan. strongly to the number of real focusing points between an ESA and a magnet, as indicated by Hintenberger and König [1]. Depending on whether this number is odd or even, either the normal or the inverse combination, respectively, must be adopted to satisfy the double-focusing condition.

2. Whether the ion beams are parallel or nonparallel before a magnet has an influence on the shape of focal plane. The double-focusing focal line is generally "straight" if the ion beams are parallel and "curved" if they are nonparallel.

These relationships are classified into four groups and shown in Figure 1 for ease of understanding. The purpose of this report is to investigate the following two subjects theoretically:

1. The direction and energy focal line of a standard Nier-Johnson-type system [2] with straight magnetic output boundary does not coincide completely for wide mass range. By giving special curvature to the magnetic output boundary calculated with the aid of computer, two focal lines can coincide completely in a first-order approximation for wide mass range (the F-N-1 system in Figure 1).

2. For a wide mass range spectrograph with a straight double-focusing focal plane, the Mattauch-Herzog [3] instrument has been the "benchmark." The purpose of this report is to design mass spectrographs with characteristics superior to the Mattauch-Herzog system (the P-N-1 system in Figure 1). 


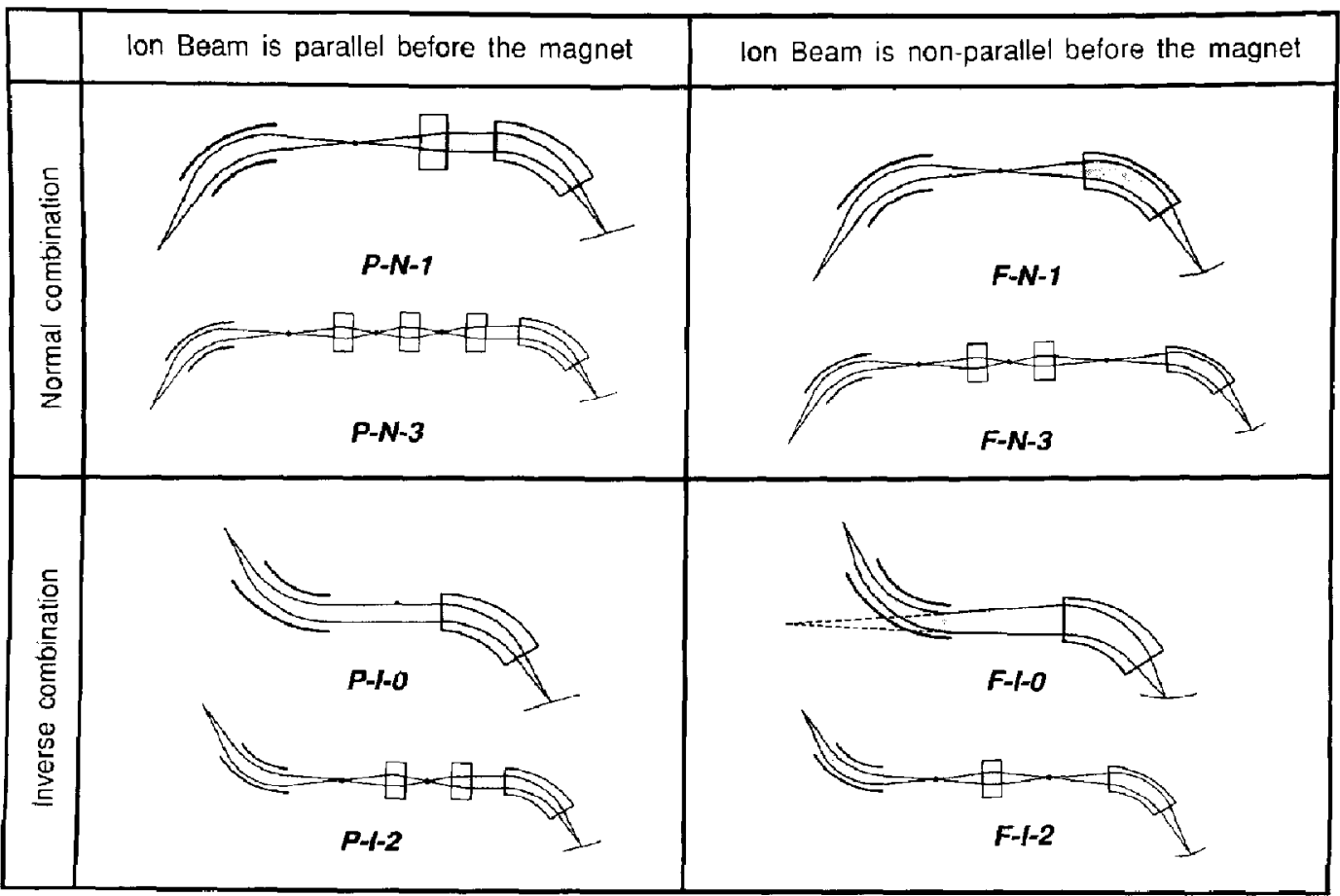

Figure 1. Classification of mass spectrographs.

\section{Ion Optical Calculation by the Matrix Method}

There are two methods of ion trajectory calculation: (1) the transfer matrix method using a computer code, for example, TRIO [4], in which an arbitrary ion is defined by its ion optical position vector $\mathbf{P}(x, \alpha, \gamma, \delta, y, \beta)$ and the field elements (electric and magnetic sectors) are expressed by transfer matrices. This method is only applicable for a narrow beam originating near the optic axis. For wide mass range application, this treatment needs to be modified. (2) The ray-trace analysis, for example, RAYTRACE [5], uses numerical expressions for the field elements derived from solutions to Maxwell's equation-the ion trajectory can then be determined by finding the solution to the path equation. In this method there are no restrictions to the beam characteristics, such as parallaxial approximations. Relating to the field calculation accuracies and computing time, the first method is preferred to the second. We have used the ray-trace method to approximate the ion optical characteristics of the new system designs and the matrix method for more precise, detailed calculations.

The coordinate system $(x, y, z)$ is defined with the origin on the optical axis, as shown in Figure 2. In principle, the ion trajectories in electric and magnetic fields can be specified in terms of an ion optical position vector $\mathbf{P}(x, \alpha, \gamma, \delta, y, \beta)$, where $x$ and $y$ are the coordinates of the ion, $\alpha$ and $\beta$ are the horizontal and vertical divergence angles, and $\gamma$ and $\delta$ are the relative mass and energy deviations, respectively. The ion optical position vector for any arbitrary position is a function of the initial vector $P_{0}\left(x_{0}, \alpha_{0}, \gamma, \delta, y_{0}, \beta_{0}\right)$. In practice, exact analytical solutions can only be obtained in certain special circumstances, and power series expansions are the normal way to express results:

$$
\begin{aligned}
& x=F_{x} x_{0}+F_{\alpha} \alpha_{0}+F_{\gamma} \gamma+F_{\delta} \delta+F_{x x} x_{0}^{2}+F_{x \alpha} x_{0} \alpha_{0} \\
& \text { (1st-order terms) } \\
& +\cdots+F_{x x x} x_{0}^{3}+F_{x x \alpha} x_{0}^{2} \alpha_{0}+\cdots \\
& \text { (3rd-order terms) } \\
& \alpha=F_{x}^{\prime} x_{0}+F_{u}^{\prime} \alpha_{0}+F_{r}^{\prime} \gamma+F_{\delta}^{\prime} \delta+F_{x x}^{\prime} x_{0}^{2}+F_{x u}^{\prime} x_{0} \alpha_{0} \\
& +\cdots+F_{x x x}^{\prime} x_{0}^{3}+F_{x x a}^{\prime} x_{0}^{2} \alpha_{0}+\cdots \\
& y=F_{y} y_{0}+F_{\beta} \beta_{0}+F_{y x} y_{0} x_{0}+F_{y \alpha} y_{0} \alpha_{0}+\cdots \\
& \text { (1st-order terms) (2nd-order terms) } \\
& \beta=F_{y}^{\prime} y_{0}+F_{\beta}^{\prime} \beta_{0}+F_{y x}^{\prime} y_{0} x_{0}+F_{y \alpha}^{\prime} y_{0} \alpha_{0}+\cdots
\end{aligned}
$$

where $F_{i j}$ are the "transfer coefficients" of the field under consideration and can be calculated numerically by using the TRIO program [4], for example. Equation 1a-1d can be expressed as a $4 \times 4$ matrix [ $F$ ] for the 


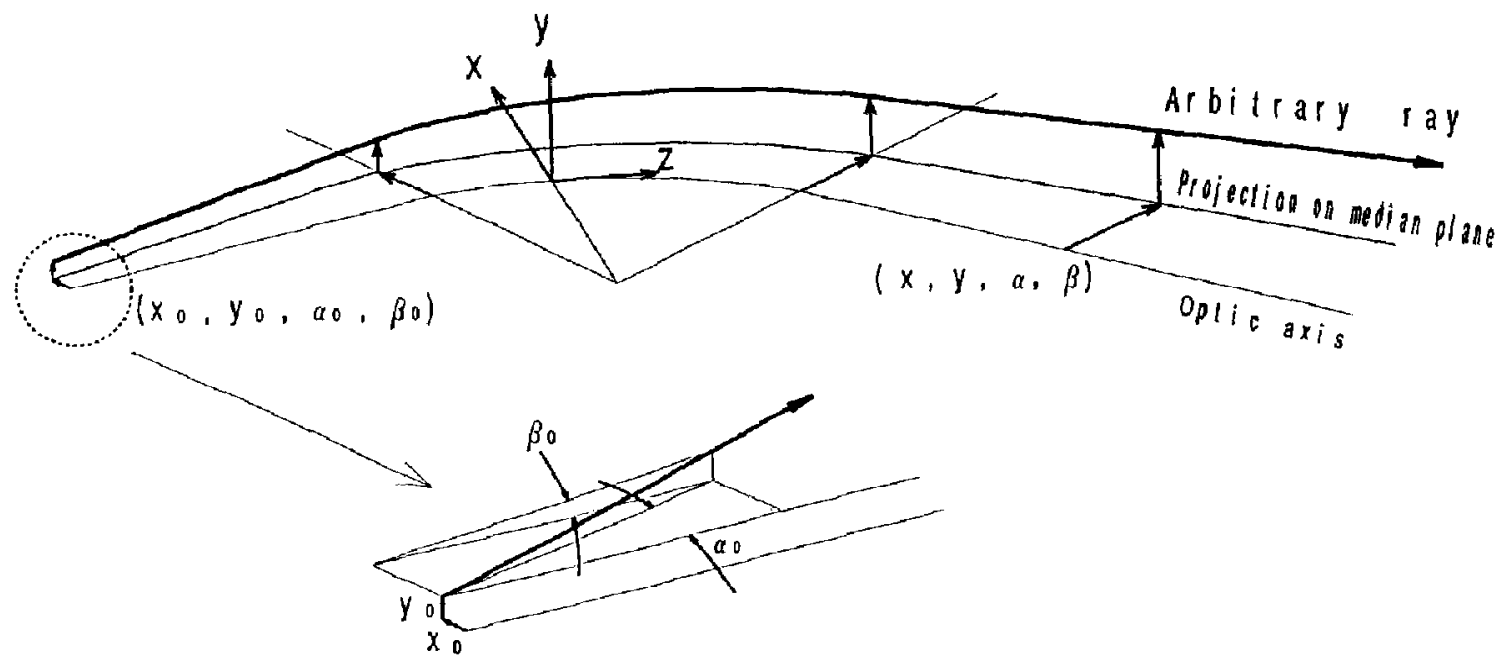

Figure 2. Definition of the coordinate system and the elements of ion optical position vectors.

first-order horizontal approximation,

$$
\left(\begin{array}{c}
x \\
\alpha \\
\gamma \\
\delta
\end{array}\right)=\left(\begin{array}{cccc}
\Gamma_{\alpha} & F_{\alpha} & F_{\gamma} & F_{\delta} \\
F_{z}^{\prime} & F_{\alpha}^{\prime} & F_{\gamma}^{\prime} & F_{\delta}^{\prime} \\
0 & 0 & 1 & 0 \\
0 & 0 & 0 & 1
\end{array} \mid\left(\begin{array}{c}
x_{0} \\
\alpha_{0} \\
\gamma \\
\delta
\end{array}\right)\right.
$$

and a $2 \times 2$ matrix in the vertical direction,

$$
\left(\begin{array}{l}
y \\
\beta
\end{array}\right)=\left|\begin{array}{ll}
F_{y} & F_{\beta} \\
F_{y}^{\prime} & F_{\beta}^{\prime}
\end{array}\right|\left(\begin{array}{l}
y_{0} \\
\beta_{0}
\end{array}\right)
$$

This is the "transfer matrix," which is used to express the characteristics of a field and is also useful in the case where several fields are combined. The ion optical character of a mass spectrometer/spectrograph can be condensed into an overall transfer matrix $[T]$, which can be obtained as the product of individual transfer mlatrices.

\section{Expression of the Overall Transfer Matrix}

Usually, the overall transfer matrix is created by multiplying the element matrices, starting at the origin. Here, we propose to parenthesize each transfer matrix into three groups- $[A],[B]$, and $[C]$, along with $[T]$, as shown in Figure 3 . The matrices $[C],[B],[A],[T]$,

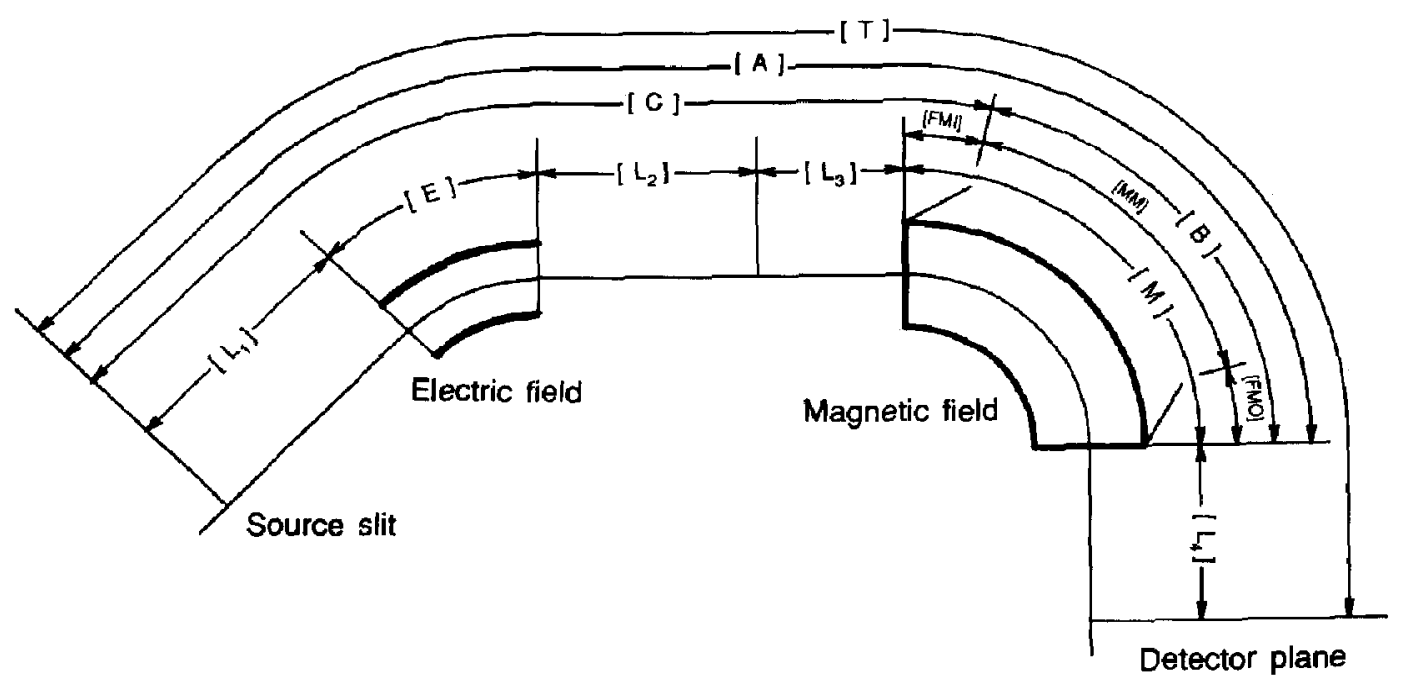

Figure 3. Definition of the transfer matrices. 
and $[M]$ are defined as

$$
\begin{aligned}
{[C] } & =[F M I]\left[L_{3}\right]\left[L_{2}\right][E]\left\lfloor L_{1}\right] \\
{[B] } & =[F M O][M M] \\
{[A] } & =[B][C] \\
{[T] } & =\left[L_{4}\right][A] \\
{[M] } & =[F M O][M M][F M I]=[B][F M T]
\end{aligned}
$$

where matrices [FMI], [MM], and [FMO] are the entrance fringing field, main field, and exit fringing field of a magnet, respectively, and matrix [E] the electric field parts, including the fringing fields:

$$
\begin{aligned}
& {[T]-\left[L_{4}\right][B][C]=\left[L_{4}\right][A]} \\
& =\left|\begin{array}{cccc}
1 & L_{4} & 0 & 0 \\
0 & 1 & 0 & 0 \\
0 & 0 & 1 & 0 \\
0 & 0 & 0 & 1
\end{array}\right|\left|\begin{array}{cccc}
B_{x} & B_{\mathbf{a}} & B_{\gamma} & B_{\delta} \\
B_{x}^{\prime} & B_{\alpha}^{\prime} & B_{\gamma}^{\prime} & B_{\delta}^{\prime} \\
0 & 0 & 1 & 0 \\
0 & 0 & 0 & 1
\end{array}\right| \\
& \times\left|\begin{array}{cccc}
C_{x} & C_{\alpha} & C_{\gamma} & C_{\delta} \\
C_{x}^{\prime} & C_{x}^{\prime} & C_{\gamma}^{\prime} & C_{\delta}^{\prime} \\
0 & 0 & 1 & 0 \\
0 & 0 & 0 & 1
\end{array}\right| \\
& =\left|\begin{array}{cccc}
1 & L_{4} & 0 & 0 \\
0 & 1 & 0 & 0 \\
0 & 0 & 1 & 0 \\
0 & 0 & 0 & 1
\end{array}\right|\left|\begin{array}{cccc}
A_{x} & A_{\alpha} & A_{\gamma} & A_{\delta} \\
A_{x}^{\prime} & A_{\gamma \gamma}^{\prime} & A_{\gamma}^{\prime} & A_{\delta}^{\prime} \\
0 & 0 & 1 & 0 \\
0 & 0 & 0 & 1
\end{array}\right| \\
& =\left|\begin{array}{cccc}
A_{x}+L_{4} A_{x}^{\prime} & A_{\alpha}+L_{4} A_{\alpha}^{\prime} & A_{\gamma}+L_{4} A_{\gamma}^{\prime} & A_{\delta}+L_{4} A_{\delta}^{\prime} \\
A_{x}^{\prime} & A_{\alpha}^{\prime} & A_{\gamma}^{\prime} & A_{\delta}^{\prime} \\
0 & 0 & 1 & 0 \\
0 & 0 & 0 & 1
\end{array}\right|
\end{aligned}
$$

\section{"SENSE" Matrix}

There are two ways of combining several fields in a series, namely, the case in which the ion deflections are in the same direction (normal combination) and that in which the ion deflections are in opposite directions (inverse combination). In the latter case, we may introduce the transfer matrix SENSE in the horizontal direction, which alters the direction of the beam, as illustrated in Figure 4. By inserting the SENSE matrix before and after the $[F]$ matrix as $[S][F][S]$ and treating this as the $[E]$ matrix, the coordinate direction can be kept constant, unrelated to the normal or inverse combination. The elements of the [S] matrix in the horizontal direction are

$$
[S]=\left|\begin{array}{rrrr}
-1 & 0 & 0 & 0 \\
0 & -1 & 0 & 0 \\
0 & 0 & 1 & 0 \\
0 & 0 & 0 & 1
\end{array}\right|
$$

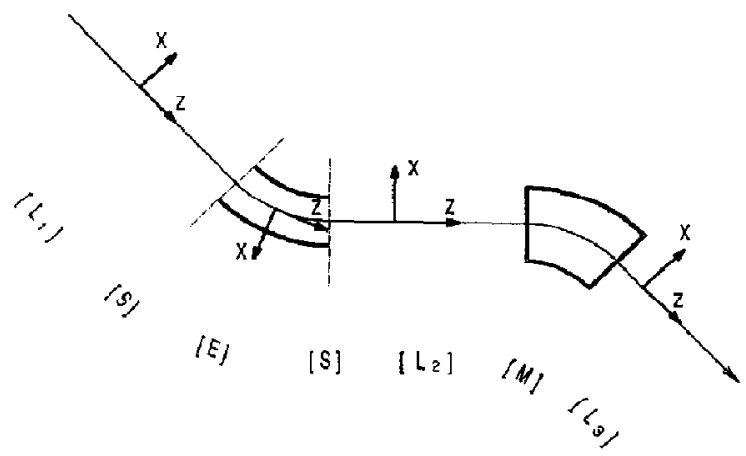

Figure 4. Direction of the $x$ and $z$ coordinates. The direction of the $y$ coordinate is always perpendicular to the $x-z$ plane. The positive direction of the $x$ coordinate is changed by multiplying the "SENSE" matrix.

\section{Double-Focusing Condition and Magnet Exit Boundary}

\section{Formation of an Intermediate Image $\left(C_{\alpha}^{\prime} \neq 0\right)$}

The distances between the magnet exit boundary and the direction focusing point $\left(L_{4 a}\right)$ and the energy focusing point $\left(L_{4 d}\right)$ can be expressed by the elements of the transfer matrix [ $A$ ] from eq $4 \mathrm{~d}$ as follows:

$$
\begin{aligned}
& L_{4 a}=-A_{\alpha} / A_{\alpha}^{\prime} \\
& L_{4 d}=-A_{\delta} / A_{\delta}^{\prime}
\end{aligned}
$$

Double focusing is achieved when $L_{4 a}=L_{4 d}$, namely,

$$
A_{\alpha} A_{\delta}^{\prime}-A_{\delta} A_{\alpha}^{\prime}=0
$$

By introducing the relevant elements of the $[B]$ and [C] matrices into eq 7 , we obtain the following double-focusing condition:

$$
\left|\begin{array}{ll}
C_{\alpha} & C_{\delta} \\
C_{\alpha}^{\prime} & C_{\delta}^{\prime}
\end{array}\right|+C_{\kappa}\left|\begin{array}{ll}
B_{x} & B_{\delta} \\
B_{x}^{\prime} & B_{\delta}^{\prime}
\end{array}\right|+C_{\alpha}^{\prime}\left|\begin{array}{ll}
B_{\alpha} & B_{\delta} \\
B_{\alpha}^{\prime} & B_{\delta}^{\prime}
\end{array}\right|=0
$$

This double-focusing condition can be also expressed by the more familiar formula using the $[E]$ and $[M]$ matrix elements of eq 3 as follows:

$$
\begin{aligned}
E_{\delta}+L_{2} E_{\delta}^{\prime}= & L_{3}\left(M_{x} M_{\delta}^{\prime}-M_{\delta} M_{x}^{\prime}\right) \\
& +\left(M_{\alpha} M_{\delta}^{\prime}-M_{\delta} M_{\alpha}^{\prime}\right)
\end{aligned}
$$

Now we are able to determine the exit boundary line for the magnetic field by following the method described by Mattauch and Herzog [3]. Two new parameters ( $d$ and $\theta$ ) need to be defined (Figure 5), and their simple relationship to $\phi_{\mathrm{m}}$ and $r_{\mathrm{m}}$ is expressed as follows:

$$
\begin{aligned}
& \theta=\phi_{\mathrm{m}} / 2 \\
& d=2 r_{\mathrm{m}} \sin \theta
\end{aligned}
$$


where $\phi_{\mathrm{m}}$ is the deflection angle, and $r_{\mathrm{m}}$ is the radius of the main optic axis. We have restricted our studies to homogeneous fields for simplicity. Then, the firstorder elements of matrix $[B]$ are expressed as

$$
\left|\begin{array}{cccc}
B_{x} & B_{\alpha} & B_{\gamma} & B_{\delta} \\
B_{x}^{\prime} & B_{\alpha}^{\prime} & B_{\gamma}^{\prime} & B_{\delta}^{\prime} \\
0 & 0 & 1 & 0 \\
0 & 0 & 0 & 1
\end{array}\right|
$$

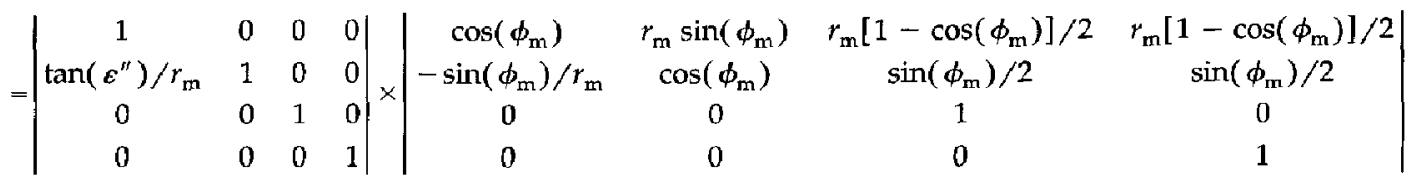

where $\quad B_{x}=\cos \left(\phi_{\mathrm{m}}\right) ; \quad B_{\alpha}=r_{\mathrm{m}} \sin \left(\phi_{\mathrm{m}}\right) ; \quad B_{\gamma}=B_{\delta}=$ $r_{\mathrm{m}}\left[1-\cos \left(\phi_{\mathrm{m}}\right)\right] / 2 ; \quad B_{x}^{\prime}=\tan \left(\varepsilon^{\prime \prime}\right) \cos \left(\phi_{\mathrm{m}}\right) / r_{\mathrm{m}}-\sin$ $\left(\phi_{\mathrm{m}}\right) / r_{\mathrm{m}} ; \quad B_{\alpha}^{\prime}=\tan \left(\varepsilon^{\prime \prime}\right) \sin \left(\phi_{\mathrm{m}}\right)+\cos \left(\phi_{\mathrm{m}}\right) ; \quad B_{\gamma}^{\prime}=$ $B_{\delta}^{\prime}=\tan \left(\varepsilon^{\prime \prime}\right)\left[1-\cos \left(\phi_{\mathrm{m}}\right)\right] / 2+\sin \left(\phi_{\mathrm{m}}\right) / 2$. By introducing eq $10 a$ and $b$ into eq 11 , we obtain

$$
\begin{aligned}
& B_{x} B_{\delta}^{\prime}-B_{\delta} B_{x}^{\prime}=\sin \theta \cos \theta \\
& B_{\alpha} B_{\delta}^{\prime}-B_{\delta} B_{\alpha}^{\prime}=r_{\mathrm{m}}\left[1-\cos \phi_{\mathrm{m}}\right] / 2=d(\sin \theta) / 2
\end{aligned}
$$

By introducing eq $12 a$ and $b$ into eq 8 , we obtain an equation that expresses the required boundary line:

$$
d=-2\left[C_{\alpha} C_{\delta}^{\prime} / C_{\alpha}^{\prime}-C_{\delta}\right] / \sin \theta-2\left(C_{\alpha} / C_{\alpha}^{\prime}\right) \cos \theta
$$

Note that the influence of oblique incidence into the magnetic field is taken into account in matrix [C], and the oblique exit angle $\varepsilon^{\prime \prime}$ has no influence on the focusing condition.

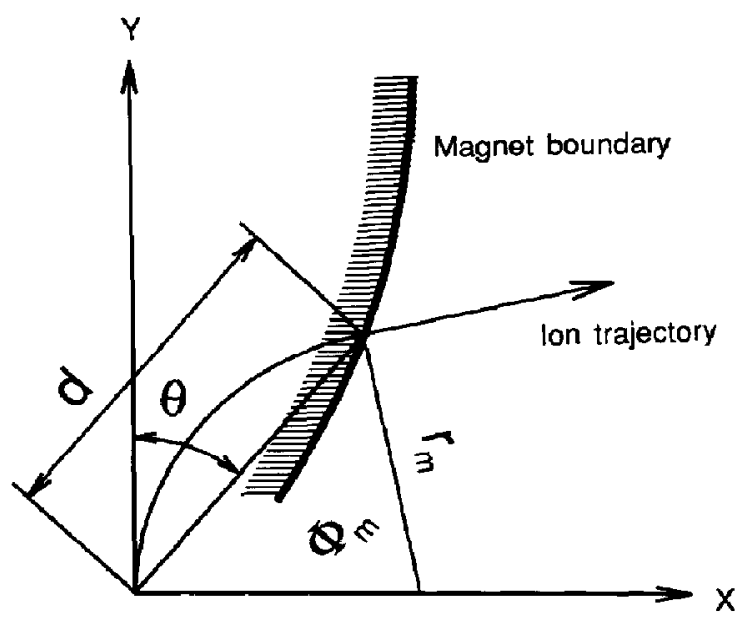

Figure 5. Coordinate systems for pole boundary.
Parallel Ion Beam Before the Magnetic. Field $\left(C_{\alpha}^{\prime}=0\right)$

We now consider the cases where the ion beam is parallel between the electric and magnetic fields (i.e., the source slit is located at the focusing point of $[E]$ such that the transfer coefficient $C_{n}^{\prime}=0$ ). The doublefocusing condition is therefore simplified as follows:

$$
\left|\begin{array}{ll}
C_{\alpha} & C_{\delta} \\
C_{\alpha}^{\prime} & C_{\delta}^{\prime}
\end{array}\right|+C_{\alpha}\left|\begin{array}{ll}
B_{x} & B_{\delta} \\
B_{x}^{\prime} & B_{\delta}^{\prime}
\end{array}\right|=0
$$

This condition can be also expressed in a more familiar form as

This expression is independent of $r_{\mathrm{m}}$ and $\phi_{\mathrm{m}}$, and therefore the image formation curve is the same for all masses. Thus, the required boundary line at the exit from the magnetic field is a straight line,

$$
\theta=\phi_{\mathrm{m}} / 2
$$

and the exit angle from the magnet $\left(\varepsilon^{\prime \prime}\right)$ is given by

$$
\varepsilon^{\prime \prime}=\phi_{\mathrm{m}} / 2-\pi / 2
$$

and the change in deflection of the ion beam is taken into account by sandwiching matrix $[E]$ with the SENSE matrix $[S]$. The double-focusing point is located at the position $L_{4}=-A_{\alpha} / A_{\alpha}^{\prime}=-A_{\delta} / A_{\delta}^{\prime}$.

\section{Designs for Mass Spectrographs}

\section{Systems Where an Intermediate Image Is Formed Before a Magnet}

This design is the standard style of a sector-type double-focusing mass spectrometer of Nier-Johnson geometry. The direction and energy focal planes generally coincide only at one point; however by shaping the exit boundary of a magnet, as shown in eq 13, two focal planes coincide completely for wide mass range in the first-order approximation. Using com-

$$
E_{\delta}^{\prime}=-\left(M_{x} M_{\delta}^{\prime}-M_{\delta} M_{x}^{\prime}\right)
$$


puter graphics facilities, it is possible to represent the magnetic field boundary (from eq 13) and the focal plane (from eq 6a and b) pictorially. As an example, the focal plane of the original [2] and modified Nier-Johnson geometries are shown in Figure $6 a, b$, and the ion optical characteristics are presented in the first two lines of Tables $1-3$. From Figure 6 , we can easily see the improvements in the focal planes for the wide mass range. It should be emphasized that any double-focusing mass spectrometer can become a wide-mass-range mass spectrograph simply by changing the shape of the exit boundary of the magnet. The principal disadvantage with this simplified approach is that the focal plane is usually curved. It is anticipated that with recent developments in array detectors, curved simultaneous detectors will soon become practicable, thus increasing the usefulness of the modified mass spectrometer/spectrograph instrument.

\section{Systems Where an Intermediate Image Is Formed at} the Entrance Boundary of a Magnet

If an intermediate image is formed at the entrance boundary of a magnet that is a straight line, as determined by eqs 16 and 17 , the direction focal plane becomes straight, and $L_{4 . A}$ is simply

$$
L_{4 A}=r_{\mathrm{m}} \sin \left(\phi_{\mathrm{m}}\right)
$$

This system will be useful as a single-focusing mass spectrograph. The merit of this system is that it has a longer distance between the magnet boundary and the focal plane than the system in which ion beams are parallel before a magnet. The relationship between this distance $L_{4}$ and the deflection angle $\phi_{\mathrm{m}}$ is shown later in Figure 9 (curve e).

\section{Inverse System Where the Ion Beam Is Parallel Before the Magnet}

The original Mattauch-Herzog geometry instruments [3] are prime examples of wide-range mass spectrographs, but they have some disadvantages:

1. The deflection angle of the electric sector $\left(\phi_{\mathrm{e}}\right)$ is unique for a given magnet and a given radius of an
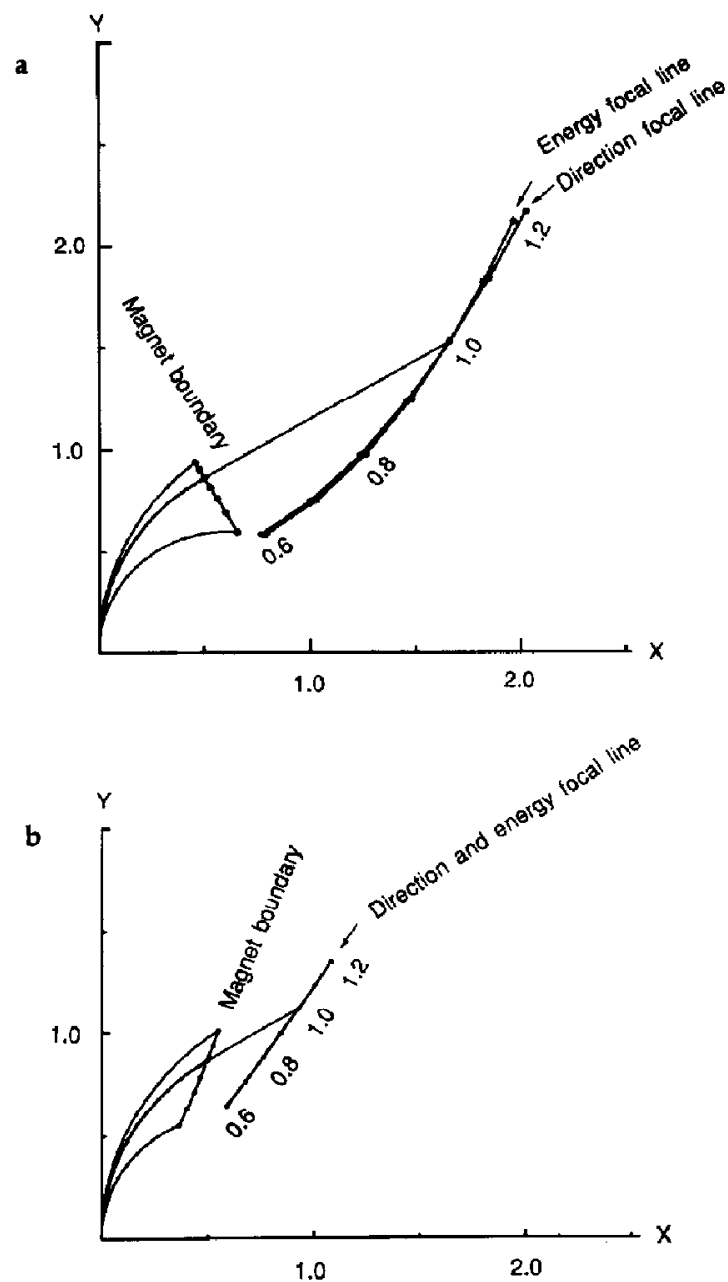

Figure 6. Focal planes and magnet boundary of mass spectrographs: (a) original Nier-Johnson; (b) modified Nier-Johnson. Numbers shown along focal line are the radii of five different ions.

electric sector $\left(r_{\mathrm{e}}\right)$ such that it is not possible to control image magnification.

2. Larger second-order image aberrations arise, especially in the vertical direction, except for the case of $\phi_{\mathrm{m}}=90^{\circ}$ with $\varepsilon^{\prime}=0$.

Table 1. Ion optical parameters of Nier-Johnson- and Mattauch-Herzog-type mass spectrometers ${ }^{\text {a }}$

\begin{tabular}{|c|c|c|c|c|c|c|c|c|c|c|c|c|c|c|c|c|}
\hline No. & $r_{e}$ & $\phi_{\mathbf{e}}$ & $R_{1 \mathrm{~g}}$ & $R_{2 e}$ & $r_{m}$ & $\phi_{\mathrm{m}}$ & $\varepsilon^{\prime}$ & $\varepsilon^{\prime \prime}$ & $R_{1 \mathrm{~m}}$ & $R_{2 m}$ & $L_{1}$ & $L_{2}$ & $L_{3}$ & $L_{4}$ & $L_{5}$ & $L_{6}$ \\
\hline 1 & 1.238 & 90.0 & 0.00 & 0.00 & 1.000 & 60.0 & 0.0 & 0.0 & 0.00 & 0.00 & 0.4380 & 0.4380 & 2.3092 & 1.3473 & 0 & 0 \\
\hline 2 & 1.238 & 90.0 & 0.00 & 0.00 & 1.000 & 59.8 & 0.0 & -51.3 & -4.80 & 6.23 & 0.4380 & 0.4380 & 2.3176 & 0.4972 & 0 & 0 \\
\hline 3 & 1.000 & 34.5 & 0.00 & 0.00 & 1.500 & 60.0 & 22.0 & -60.0 & -5.00 & 0.00 & 0.6600 & 0 & 0 & 0.5920 & 0 & 1.0000 \\
\hline 4 & 1.000 & 42.8 & 0.00 & 0.00 & 1.200 & 75.0 & 20.4 & -52.5 & -3.00 & 0.00 & 0.4251 & 0 & 0 & 0.4397 & 0 & 1.0000 \\
\hline 5 & 1.000 & 31.5 & 0.00 & 0.00 & 1.000 & 90.0 & 0.0 & -45.0 & 0.00 & 0.00 & 0.7702 & 0 & 0 & 0.0000 & 0 & 1.0000 \\
\hline 6 & 1.000 & 90.0 & 0.00 & 0.00 & 1.200 & 75.0 & 20.4 & -52.5 & -3.00 & 0.00 & 0.8000 & 0.0610 & 0.1390 & 0.4397 & 0.7153 & 1.0000 \\
\hline
\end{tabular}

\footnotetext{
"Farameters are defined in Figure 7 . For Nos. 1 and 2, lengths $L_{91}, L_{5}, L_{92}$, and $L_{6}$ are set at zero; for Nos. $3-5$, lengths $L_{2}, L_{3}, L_{97}, L_{5}$, and $L_{q 2}$ are set at zero; for No. 6 , the length and field strength of two quadrupole lenses are $L_{q 1}=L_{q 2}=0.25$ and $Q_{k 1}=$ 10.5551 and $Q_{k 2}=2.1721$, respectively. The direction of deflection of an ESA and a magnet is normal for Nos. 1 and 2 and inverse for Nos. 3-6.
} 
Table 2. First- and second-order overall transfer matrix elements for Nier-Johnson- and Mattauch-Herzog-type mass spectrometers.

\begin{tabular}{|c|c|c|c|c|c|c|c|c|c|c|c|c|}
\hline No. & $T_{x}$ & $T_{\alpha}$ & $\tau_{\gamma}$ & $T_{\delta}$ & $T_{y}$ & $T_{\beta}$ & $T_{\alpha \alpha}$ & $T_{\alpha \delta}$ & $T_{\delta \delta}$ & $T_{y y}$ & $T_{y \beta}$ & $T_{\delta \delta}$ \\
\hline 1 & 0.667 & 0.000 & 0.833 & 0.000 & 1.088 & 8.008 & 0.084 & 0.023 & 0.290 & -0.859 & -9.919 & -28.842 \\
\hline 2 & 0.247 & 0.000 & 0.308 & 0.000 & 1.750 & 11.258 & 0.195 & 0.623 & -0.770 & 0.216 & 0.956 & -0.974 \\
\hline 3 & -0.712 & 0.000 & 0.374 & 0.000 & 0.911 & 5.433 & $1: 744$ & -0.622 & 0.947 & 0.398 & 0.507 & -0.994 \\
\hline 4 & -0.855 & 0.000 & 0.444 & 0.000 & 0.711 & 4.386 & 0.619 & 1.398 & -0.277 & 0.438 & 0.468 & -2.378 \\
\hline 5 & -0.946 & 0.000 & 0.500 & 0.000 & 0.037 & 3.974 & 1.180 & 2.863 & -0.616 & 0.000 & -0.024 & -0.054 \\
\hline 6 & -0.349 & 0.000 & 0.444 & 0.000 & 685 & 1819 & -0.030 & -3.503 & -2.202 & a & a & a \\
\hline
\end{tabular}

a Large numbers.

3. Vertical ( $y$ directional) focusing is not satisfied in the case of the original Mattauch-Herzog design consisting of a cylindrical ESA and a homogeneous magnet. It should be noted that the improved Mattauch-Herzog system with a spherical ESA satisfied the vertical focusing [6].

Ion optical details of the Mattauch-Herzog geometry with three different deflection angles $\phi_{\mathrm{m}}$ are presented in Tables 1-3 (third to fifth lines). To overcome such restrictions, an electrostatic quadrupole lens may be introduced to satisfy the double-focusing condition with another value of $\phi_{c}$. Some examples of this are given in ref 7 .

Alternatively, it is also possible to design systems with two quadrupole lenses to form two real images between the ESA and the magnet that satisfy the criterion of double focusing for any arbitrary $\phi_{\mathrm{e}^{*}}$. An example of this is presented in Table 1 (sixth line) and illustrated in Figure 1 (P-I-2). The first-order characteristics in the principal (horizontal) focusing direction are exactly the same as the system discussed later $\left(\phi_{\mathrm{m}}=75^{\circ}\right.$ system) because the same ESA and magnet are used; however, the ion optical character in the vertical direction and the higher order aberrations differ considerably. This system is not yet suitable for practical use.

\section{Normal System Where the Ion Beam Is Parallel Before the Magnet}

Equation 14 is valid for both inverse and normal combinations of electric and magnetic sectors. In this section, we discuss normal-configuration mass spec- trographs. If the source slit is placed at the focus point of the ESA part containing the electric quadrupole in a normal configuration, then the ion beam will be parallel on exiting that sector, but the double-focusing condition cannot be satisfied because no intermediate image is formed. At least one real intermediate image (including those within the fields) must be formed between the focusing elements (see the P-N-1 system in Figure 1). Three methods for making the ion beam parallel before the magnet after producing a real image in or after the ESA have previously been proposed:

1. to form an image within the ESA with a parallel beam after the electric field [8];

2. to use two electrostatic analyzers with an image formed between the two electric sectors [9]; and

3. to use an electrostatic quadrupole lens. The exit focal point of the ESA coincides with the focal point of the quadrupole such that the ion beam is parallel after quadrupole element [10].

The principal disadvantage of these methods is the lack of consideration given to vertical focusing-this is fundamental if high transmission (sensitivity) is required. We propose the introduction of a quadrupole doublet that will have the advantage of vertical focusing as well as making the ion beam parallel before the magnet. We could then stipulate the following requirements for the new mass spectrographs:

1. high mass resolution - smaller image magnification and corrected second- and third-order aberrations;

2. introduction of a slit at the image point of the ESA to limit the energetically dispersing beam;

Table 3. Third-order matrix elements for Nier-Johnson- and Mattauch-Herzog-type mass spectrometers

\begin{tabular}{|c|c|c|c|c|c|c|c|c|c|c|}
\hline No. & $T_{\alpha \alpha \alpha}$ & $T_{\alpha \alpha \delta}$ & $T_{\alpha \delta \delta}$ & $T_{\delta \delta \delta}$ & $T_{x e p y}$ & $T_{\alpha y \beta}$ & $T_{\alpha \beta \beta}$ & $T_{\delta y \gamma}$ & $T_{\delta y \beta}$ & $T_{\delta \beta \beta}$ \\
\hline 1 & -1.489 & -2.789 & 11.141 & 0.014 & -0.401 & -8.436 & $-32,894$ & 0.645 & 3.757 & -2.121 \\
\hline 2 & 120.225 & -393.533 & 431.810 & -154.120 & 4.984 & 92.843 & 366.234 & -6.441 & -108.638 & -413.396 \\
\hline 3 & -14.875 & 30.018 & -36.164 & 6.932 & -0.458 & -10.929 & -44.407 & -3.262 & -6.611 & 25.023 \\
\hline 4 & -9.385 & 14.436 & -19.848 & 8.058 & -2.425 & -17.535 & -35.154 & -0.376 & 2.861 & 21.628 \\
\hline 5 & -29.076 & 3.909 & -0.998 & 1.329 & -0.529 & -2.479 & -5.841 & 0.560 & 2.123 & 3.154 \\
\hline 6 & -44.169 & -351.796 & -806.378 & -579.479 & a & a & a & $a$ & a & a \\
\hline
\end{tabular}

\footnotetext{
a Large numbers.
} 
3. a degree of vertical focusing for high sensitivity; and

4. straight focal plane apart from the magnet boundary for a wide mass range.

A block diagram of the system investigated and its parameters are shown in Figure 7. By using the quadrupole doublet, double focusing for all masses and stigmatic focusing for certain masses can be fulfilled for almost any type of electric and magnetic field to a first-order approximation. Because the ion beam is "parallel" between the electric and magnetic fields, the sector characters may be considered independently.

First-Order Parameters of the Magnet. Five parameters define the first-order character of a magnetic field: $r_{m}$ is the radius of the main optic axis, $\phi_{m}$ the deflection angle, $n_{1}$ the field constant, $\varepsilon^{\prime}$ the incident angle, and $\varepsilon^{\prime \prime}$ the exit angle. In this report, only homogeneous magnets are considered $\left(n_{1}=0\right)$. The first important consideration is that of mass resolution, which is defined as $T_{\delta} / T_{x}$, where $T_{\delta}$ and $T_{x}$ are the mass dispersion and image magnification, respectively. It should be noted that by increasing the deflection angle $\phi_{\mathrm{m}}$ and/or the radius of the main optic axis $r_{m}$, the image magnification $T_{x}$ and mass dispersion coefficients $T_{\delta}$ are increased proportionally; however, the factor $T_{\delta} / T_{x}$, which is proportional to the theoretical mass resolution, remains unchanged if the same electric field is used. If a channel plate is used as a position-sensitive detector, then the overall spacial resolution is approximately $50 \mu \mathrm{m}$, so the image on the focal plane needs to be of the order of several hundred micrometers to maintain mass accuracy.

The second important point is that of mass range, which is directly related to the radius of the ion trajectory in the magnet $r_{\mathrm{m}}$. If the effective size of the magnet yoke is fixed, the product $\left(r_{\mathrm{m}} \cdot \phi_{\mathrm{m}}\right)$ is nearly determined, and it must be decided which parameter has priority within a study. Here, we consider four cases $\left(\phi_{\mathrm{m}}=45^{\circ}, r_{\mathrm{m}}=2.0 ; \phi_{\mathrm{m}}=60^{\circ}, r_{\mathrm{m}}=1.5 ; \phi_{\mathrm{m}}=\right.$ $75^{\circ}, r_{\mathrm{m}}=1.2 ; \phi_{\mathrm{m}}=90^{\circ}, r_{\mathrm{m}}=1.0$ ) as typical examples, although any arbitrary value of $\phi_{\mathrm{m}}$ can be used.

When a photographic plate was used as the detector, the system with $L_{\ddagger a}=0$ was preferred because the plates were free from major influences caused by the magnetic fringing field. When electronic (channel plate) detectors are used, it is advisable to locate them as far away from the magnet boundary as possible because the gain of a channel plate decreases, owing to the influence of the magnetic field. This brings us to the third important factor, the angle of separation $\boldsymbol{w}_{f}$ between the detector and the exit boundary of the magnet. The relationship between the angle of separation $\omega_{\mathrm{f}}$ and the deflection angle $\phi_{\mathrm{m}}$, choosing the angle of incidence into the magnet $\varepsilon^{\prime}$ as a parameter. is shown in Figure 8. Another way to express the degree of separation of a focal plane, which is the distance between a focus point and the magnet boundary $L_{4}$, is shown in Figure 9 . It should be noted that $\omega_{f}$

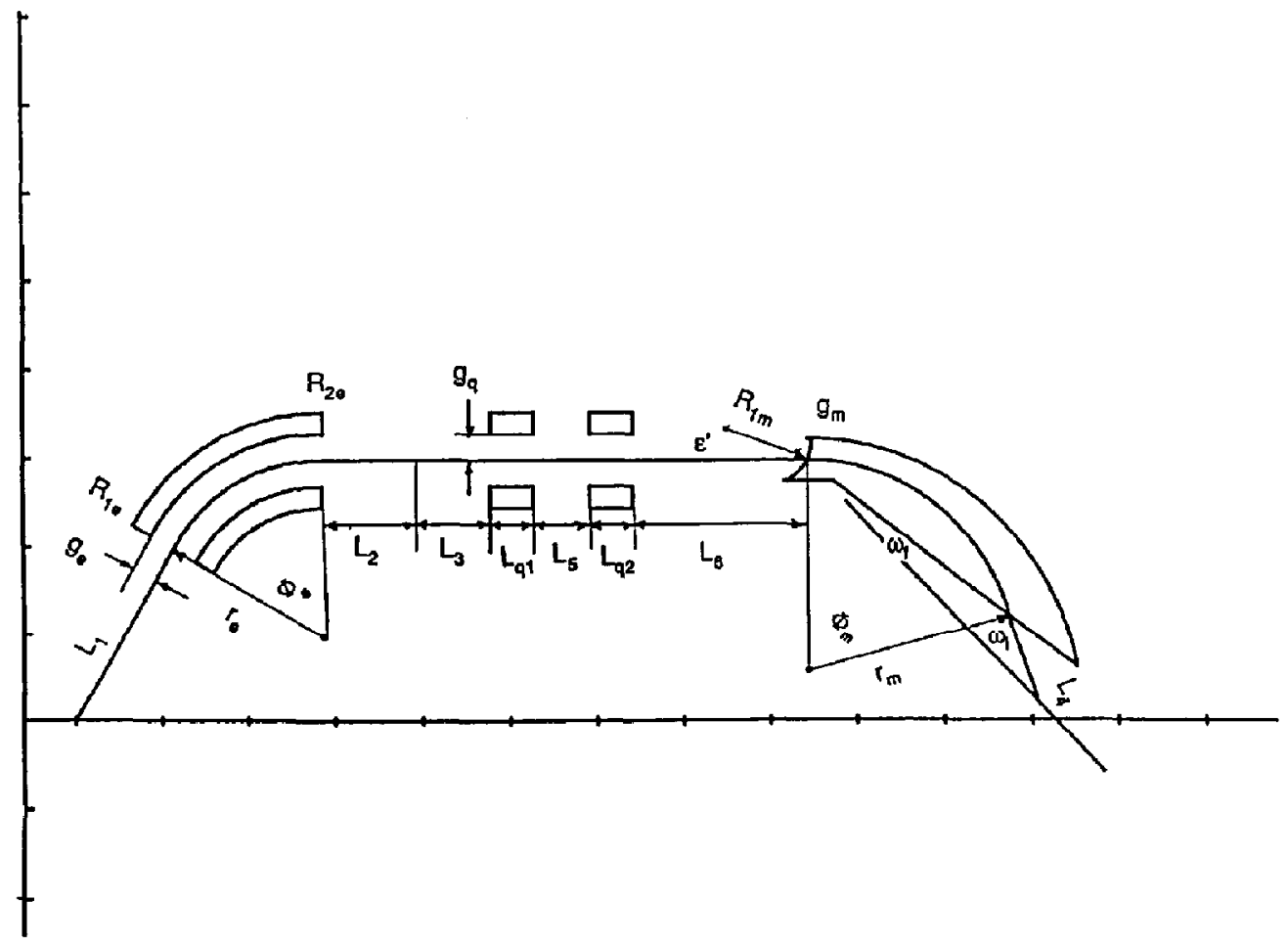

Figure 7. Definition of geometric parameters of a mass spectrograph. 


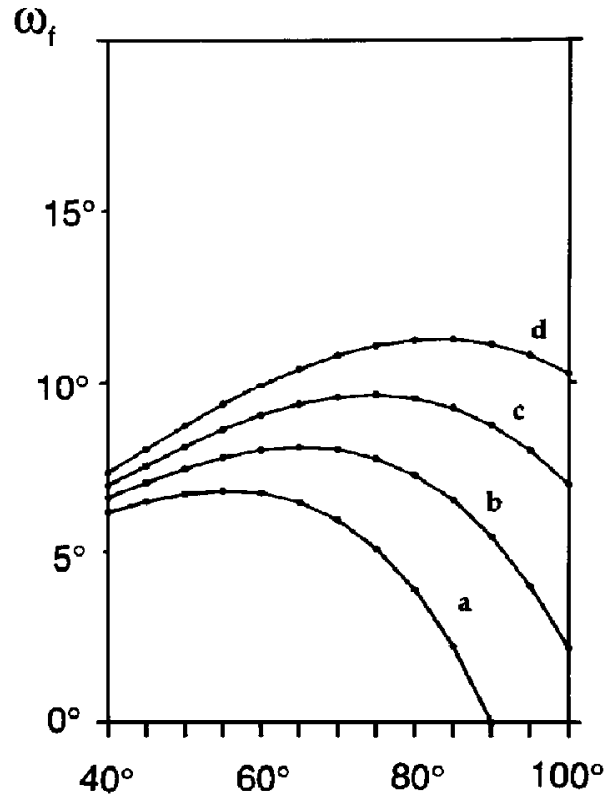

Figure 8. Relationship of the separation angle $\omega_{i}$ and magnetic deflection angle $\phi_{\mathrm{m}}$ for various $\varepsilon^{\prime}: 0^{\circ}(a), 15^{\circ}(b), 30^{\circ}(c), 45^{\circ}(d)$.

is maximized for all deflection angles at a fixed value of $\varepsilon^{\prime}$.

Fourth, the angle of incidence of the ion beam on the focal plane $\omega_{i}$ is also of concern when using channel plates. The relationship between this parame-

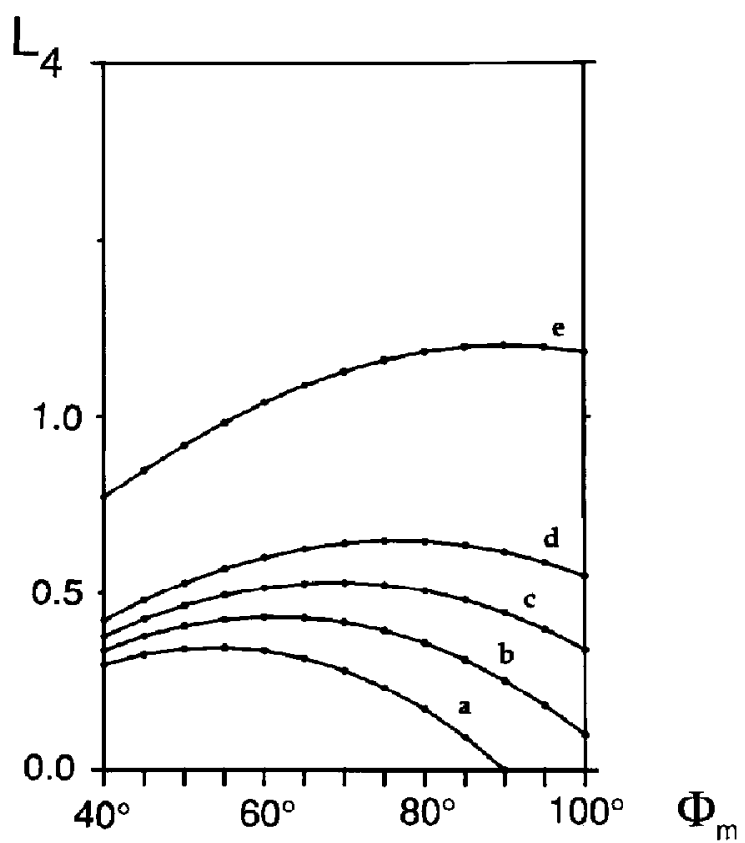

Figure 9. Relationship of focusing distance $L_{4}$ and magnetic deflection angle $\phi_{\mathrm{m}}$ for various $\varepsilon^{\prime}: 0^{\circ}(a), 15^{\circ}(b), 30^{\circ}(c), 45^{\circ}(d)$; (e) a system where the intermediate focusing is achieved at the magnetic entrance, where $r_{\mathrm{m}}=1.2$. ter and the deflection angle $\phi_{m}$ and incidence angle of the magnet $\varepsilon^{\prime}$ is illustrated in Figure 10 . With regard to $\varepsilon^{\prime \prime}$, the same relationship as shown in eq 17 should be satisfied because the ion beams are parallel before the magnet.

Second-Order Parameters of the Magnet. The radius of curvature $R_{7 \mathrm{~m}}$ of the magnet field boundary has considerable influence on second-order image aberrations, as shown in Figure 11a and is also a function of the deflection $\phi_{\mathrm{m}}$ and incidence angles of the magnet $\varepsilon^{\prime}$. The theoretical mass resolution under certain source slit conditions can be simulated by TRIO and is shown in Figure 11b. Such a graph is often helpful to evaluate any newly designed ion optical system. From the manufacturing viewpoint, the upper and lower limits of $R_{1 \mathrm{~m}}$ are limited, so we must obtain the optimum condition for the incidence angle $\varepsilon^{\prime}$ under a given $R_{1 \mathrm{~m}}$ from Figure 12. The graphs in Figure 12 remain nearly the same even if the ESA is changed and are thus very useful for designing a mass spectrograph consisting of a homogeneous magnet and any type of ESA and quadrupole doublet.

First- and Second-Order Parameters of the ESA. The electric field is limited to a cylindrical electric sector and quadrupole lenses. The field strengths of the quadrupoles are determined by TRIO-FIT with a fitting routine [11] to satisfy the condition of $C_{\alpha}^{\prime}=0$ (the condition that ion beams are parallel before a magnet)

$\omega_{i}$
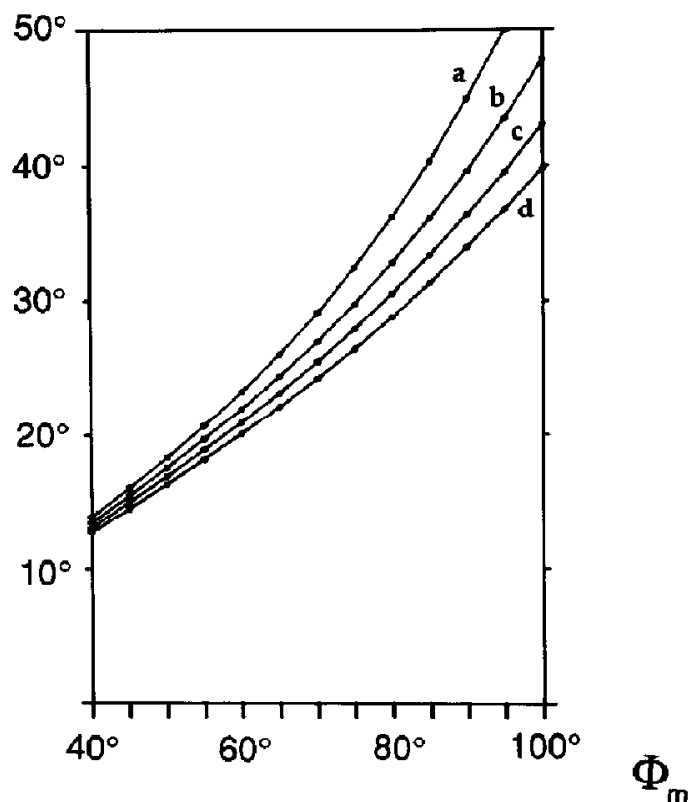

Figure 10. Relationship of the incident angle $\omega_{1}$ and the magnetic deflection angle $\phi_{\mathrm{n} 2}$ for various $\varepsilon^{\prime}: 0^{\circ}(a), 15^{\circ}(b), 30^{\circ}(c)$, $45^{\circ}(d)$. 

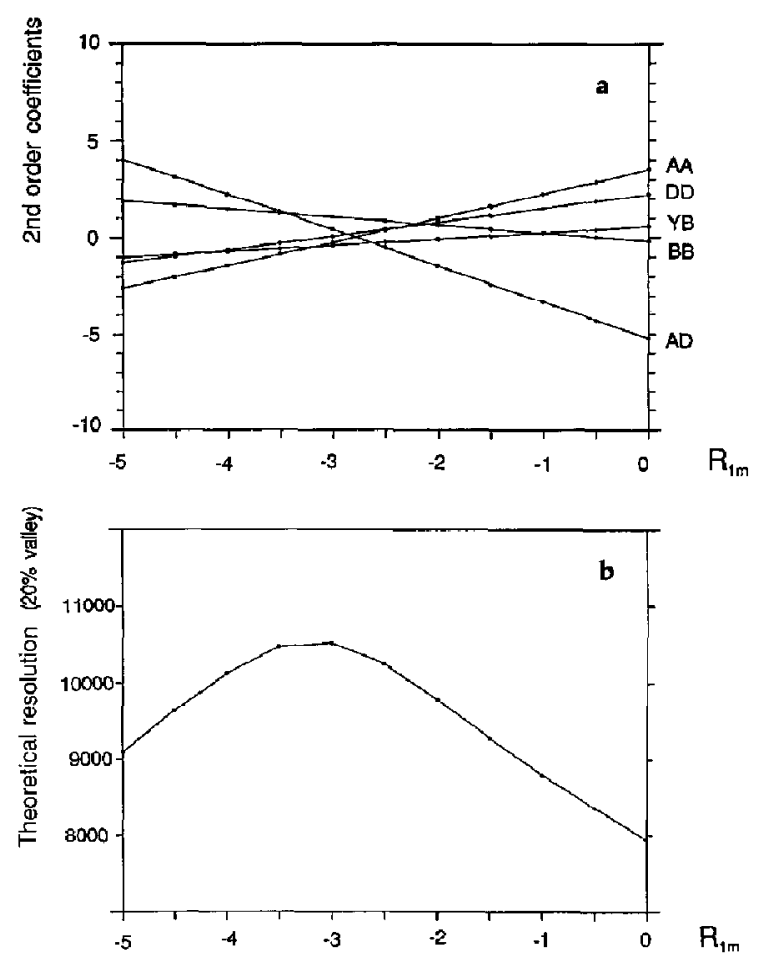

Figure 11. Influence of $R_{1 \mathrm{~m}}$ on the second-order image aberration coefficients: (a) second-order aberration coefficients; (b) calculated mass resolution, where $x_{0}=0.0001 ; \alpha_{0}=0.002 ; \delta=$ $0.002 ; y_{0}=0.001 ; \beta_{0}=0.002$.

and $T_{B}=0$ (the vertical focusing condition). The radius of the ESA $r_{e}$, the angle of deflection $\phi_{e}$, and the first drift length $L_{1}$ are selected as parameters, thus affecting the image magnification $T_{x}$, as shown in Figure 13. The mass dispersion $T_{\delta}$ remains the same if the same magnetic field is used; thus, the factor $T_{\delta} / T_{x}$ is dependent solely on the ESA. The second-order parameters $R_{16}$ and $R_{2 \text { : }}$ are determined so as to minimize the second-order aberration coefficients.

\section{Thirteen Examples of Mass Spectrographs}

In this section, we present 13 examples of wide-massrange (decade detection) mass spectrographs that are a combination of $\phi_{\mathrm{e}}=60^{\circ}, 75^{\circ}$, and $90^{\circ}$ and $\phi_{\mathrm{m}}=45^{\circ}$, $60^{\circ}, 75^{\circ}$, and $90^{\circ}$. The overall transfer matrix elements for other radii of ions with different masses are then determined using these criteria. Because the focal plane detector is planned to be attached, the maximum resolution of an instrument is therefore limited by the resolution of the focal plane detector. Then the second and third aberration eliminations are not critical. Thus, elaborate parameter combinations are not necessary, and we can limit ourselves to the consideration of 12 combinations of $\phi_{\mathrm{e}}=60^{\circ}, 75^{\circ}$, and $90^{\circ}$ and $\phi_{\mathrm{m}}=45^{\circ}$, $60^{\circ}, 75^{\circ}$, and $90^{\circ}$ as typical examples. The results are presented in Table 4 (parameters) and Tables 5 and 6

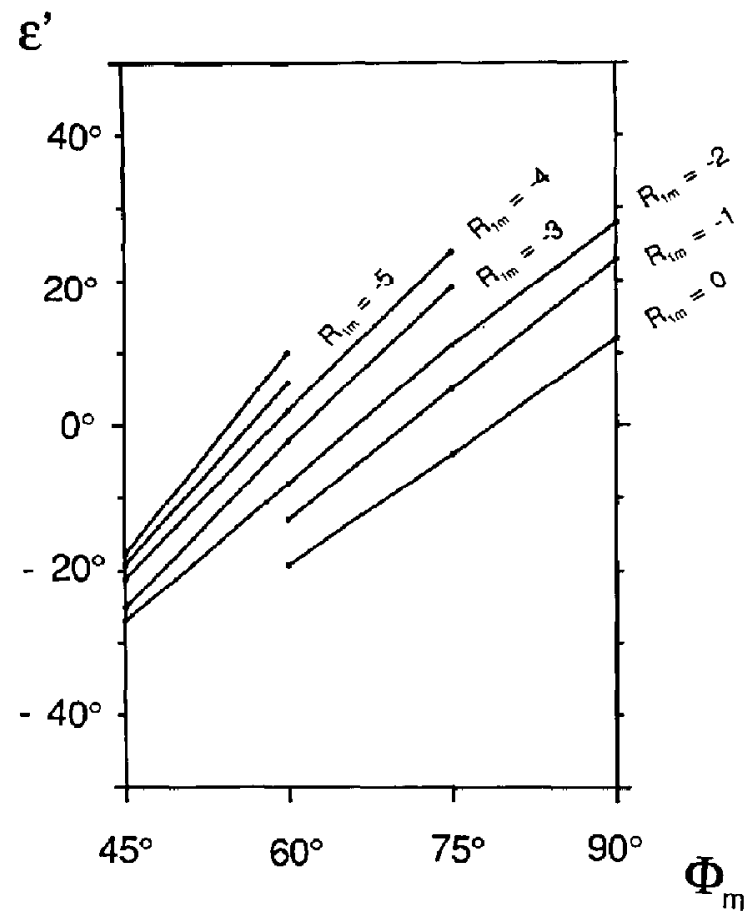

Figure 12. Relationship of $\varepsilon^{\prime}$ and $\phi_{\mathrm{m}}$ that gives the best second-order focusing condition for a given $R_{1 \mathrm{~m}}$ value.

$T_{x}$

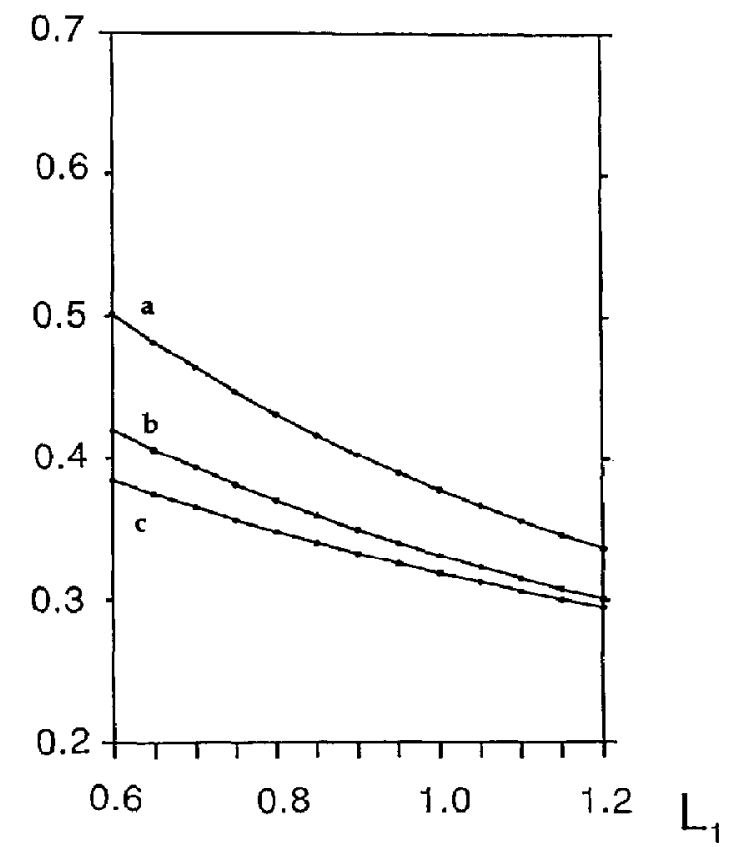

Figure 13. Relationship of overall image magnification $T_{\mathrm{x}}$ and the first drift length $L_{1}$ for a given $\phi_{\mathrm{e}}: 60^{\circ}(a), 75^{\circ}(b), 90^{\circ}(c)$. 
Table 4. Ion optical parameters of newly proposed mass spectrographs ${ }^{\mathrm{a}}$

\begin{tabular}{|c|c|c|c|c|c|c|c|c|c|c|c|c|c|c|c|c|}
\hline No. & $r_{\mathrm{e}}$ & $\phi_{0}$ & $R_{10}$ & $R_{2 e}$ & $r_{m}$ & $\phi_{\mathrm{m}}$ & $\varepsilon^{\prime}$ & $\varepsilon^{\prime \prime}$ & $\boldsymbol{R}_{1 \mathrm{~m}}$ & $R_{2 \mathrm{~m}}$ & $L_{1}$ & $L_{2}$ & $L_{3}$ & $L_{4}$ & $a_{k 1}$ & $a_{k 2}$ \\
\hline 1 & 1.00 & 60.00 & 0.00 & 5.00 & 2.00 & 45.00 & -17.80 & -67.50 & -6.00 & 0.00 & 1.140 & 0.560 & 1.253 & 0.440 & -2.062 & 1.937 \\
\hline 2 & 1.00 & 5.00 & 00 & 00 & 2.00 & 5.00 & -17.80 & -67.50 & -6.00 & 0.00 & 0.908 & 0.298 & .222 & 0.440 & -2.084 & 1.949 \\
\hline 3 & 1.00 & 90.00 & 0.00 & 5.00 & 2.00 & 45.00 & -17.80 & -67.50 & -6.00 & 0.00 & 0.800 & 0.061 & 1.203 & 0.440 & & 1.954 \\
\hline 4 & 1.00 & 60.00 & 0.00 & 5.00 & 1.50 & 60.00 & 5.80 & -60.00 & -5.00 & 0.00 & 1.140 & 0.560 & 0.652 & 0473 & 30 & 2.079 \\
\hline 5 & 1.00 & 75.00 & .00 & .00 & 1.50 & 60.00 & 80 & $-6 c$ & -5.00 & 0.00 & 08 & 298 & & 0.473 & & 2.092 \\
\hline 6 & 1.00 & & 00 & .00 & & & & & & & & & & & & \\
\hline 7 & 1.00 & 60.00 & 0.00 & 5.00 & 1.20 & 75.00 & 20.40 & -52.50 & -3.00 & 0.00 & 1.140 & 0.560 & 0.350 & 0.440 & -2.169 & 2.208 \\
\hline 8 & 1.00 & 75.00 & 0.00 & 5.00 & 1.20 & 75.00 & 20.40 & -52.50 & -3.00 & 0.00 & 0.908 & 0.298 & 0.335 & 0.440 & -2.200 & 2.221 \\
\hline 9 & 1.00 & 90.00 & 0.00 & 5.00 & 1.20 & 75.00 & & -52.50 & -3.00 & 0.00 & 0.800 & 0.061 & 0.326 & 0.440 & & 2.228 \\
\hline 10 & 1.00 & 60.00 & 0.00 & 5.00 & 1.00 & 90.00 & 28.00 & -45.00 & -2.00 & 0.00 & 1.140 & 0.560 & 0.194 & 0.346 & & 2.311 \\
\hline 11 & 1.00 & 75.00 & 0.00 & 5.00 & 1.00 & 90.00 & & -45.00 & -2.00 & 0.00 & 0.908 & 0.298 & 0.182 & 0.346 & -2.216 & 2.324 \\
\hline 12 & 1.00 & 90.00 & 0.00 & 5.00 & 1.00 & 90.00 & 28.00 & -45.00 & -2.00 & 0.00 & 0.800 & 0.061 & 0.175 & 0.346 & -2.230 & 2.331 \\
\hline 13 & 1.00 & 90.00 & 0.00 & 5.00 & 1.00 & 90.00 & 0.00 & -45.00 & 0.00 & 0.00 & 0.800 & 0.061 & 0.518 & 0.000 & -2.214 & 2.141 \\
\hline
\end{tabular}

aparameters are defined in Figure 7; the length of the two quadrupoles are $L_{q 1}$ and $L_{\mathrm{q} 2}=0.25$

Table 5. First-and second-order overall transfer matrix elements and theoretical mass resolution under the source slit condition of $x_{0}=0.0001, \alpha_{0}=0.002, \delta=0.002, y_{0}=0.001, \beta_{0}=0.002$

\begin{tabular}{|c|c|c|c|c|c|c|c|c|c|c|c|c|c|}
\hline No. & $T_{x}$ & $T_{\alpha}$ & $T_{\gamma}$ & $I_{\delta}$ & $T_{r}$ & $T_{\beta}$ & $T_{\alpha \alpha}$ & $T_{\alpha \delta}$ & $T_{\delta \hat{\delta}}$ & $T_{y \gamma}$ & $T_{y \beta}$ & $T_{\delta \delta}$ & Resolution \\
\hline 1 & 0.229 & 0.000 & 0.291 & 0.000 & -2.311 & 0.005 & -0.450 & 1.400 & -0.010 & -1.597 & -1.053 & -0.915 & 7189 \\
\hline 2 & 0.229 & 0.000 & 0.291 & 0.000 & -2.482 & -0.009 & -1.267 & 1.341 & 0.035 & -1.569 & -0.160 & -0.034 & 7501 \\
\hline 3 & 0.229 & 0.000 & 0.291 & 0.000 & -2.550 & 0.003 & -1.545 & 0.921 & 0.103 & -1.448 & 0.725 & 0.874 & 7641 \\
\hline 4 & 0.294 & 0.000 & 0.374 & 0.000 & $-2,394$ & 0,001 & 1.280 & 0.388 & 0.159 & -1.598 & -2.388 & -1.454 & 8104 \\
\hline 5 & 0.294 & 0.000 & 0.374 & 0.000 & -2.590 & 0.002 & 0.227 & 0.411 & 0.176 & -1.491 & -1.223 & -0.308 & 9115 \\
\hline 6 & 0.294 & 0.000 & 0.374 & 0.000 & -2.672 & 0.010 & -0.100 & 0.145 & 0.174 & -1.306 & -0.096 & 0.870 & \\
\hline 7 & 0.349 & 0.000 & 0.444 & 0.000 & -2.082 & 0.000 & 1.586 & 0.621 & 0.090 & -1.733 & -3.291 & -1.747 & 8461 \\
\hline 8 & 0.349 & 0.000 & 0.444 & 0.000 & -2.266 & -0.001 & 0.348 & 0.650 & 0.107 & -1.579 & -1.918 & -0.397 & 9668 \\
\hline 9 & 0.349 & 0.000 & 0.444 & 0.000 & -2.344 & 0.000 & -0.054 & 0.368 & 0.113 & -1.350 & -0.595 & 99 & 10467 \\
\hline 10 & 0.392 & 0.000 & 0.499 & 0.000 & -1.605 & 0.004 & 1.125 & 1.454 & -0.104 & -1.741 & -4.009 & -1.620 & 8342 \\
\hline 11 & 0.392 & 0.000 & 0.499 & 0.000 & -1.754 & 0.000 & -0.263 & 1.471 & -0.077 & -1.527 & -2.493 & -0.168 & 9347 \\
\hline 12 & 0.392 & 0.000 & 0.499 & 0.000 & -1.817 & 0.001 & -0.712 & 1.048 & -0.017 & -1.250 & -1.014 & 3.379 & 9969 \\
\hline 13 & 0.393 & 0.001 & 0.500 & 0.000 & -1.693 & -0.003 & -1.539 & 0.354 & 0.837 & -0.381 & -0.887 & -0.194 & 9846 \\
\hline
\end{tabular}

Table 6. Third-order matrix elements for the newly proposed mass spectrographs

\begin{tabular}{|c|c|c|c|c|c|c|c|c|c|c|c|c|}
\hline No. & $T_{\alpha u x}$ & $T_{\mathrm{au} \delta}$ & $T_{\mathbf{\alpha} \delta \delta}$ & $T_{\sigma \sigma \delta}$ & $T_{x y y y}$ & $T_{x y \beta}$ & $T_{\mathbf{\alpha} \beta \beta}$ & $T_{\delta y y}$ & $T_{\delta \mu \beta}$ & $T_{\delta \beta \beta}$ & $T_{a \gamma \gamma}$ & $T_{\gamma \gamma \delta}$ \\
\hline 1 & 725.699 & -1641.457 & 1224.419 & -301.384 & 94.753 & 130.703 & -12.188 & -24.071 & 12.596 & 21.431 & 3.044 & -2.162 \\
\hline 2 & 750.760 & -1626.986 & 1167.892 & -279.869 & 89.064 & 96.972 & -17.267 & -16.136 & 41.448 & 4.580 & 3.043 & -2.1 \\
\hline 3 & 848 & -1452.566 & 922.174 & -198.037 & 76.640 & 58.240 & -23.019 & -0.773 & 78.262 & 27.467 & 3.042 & -1.8 \\
\hline 4 & 3.325 & -460.580 & 10.498 & -118.678 & 58.943 & 91.507 & 36.677 & -11.323 & 18.901 & 1.625 & 2.265 & -1.7 \\
\hline 5 & 185.866 & -469.816 & 396.164 & -112.147 & 53.869 & 62.610 & 22.569 & -4.495 & 43. & 10.477 & 2.265 & -1.6 \\
\hline 6 & 929 & -427.457 & 322.200 & -84.364 & 43.717 & 29.427 & 10.248 & 6.955 & 74.851 & 21.648 & 2.264 & -1 \\
\hline 7 & 3.870 & -153.014 & 178.260 & -62.908 & 42.565 & 79.842 & 62.483 & -2.727 & 29.599 & -5.304 & 1.720 & -1.3 \\
\hline 8 & 52.465 & -167.253 & 173.900 & -60.311 & 36.980 & 52.586 & 44.382 & 4.023 & 53.008 & 5.650 & 1.720 & -1.310 \\
\hline 9 & 58.723 & -157.138 & 145.281 & -48.289 & 27.284 & 21.821 & 30.699 & 13.944 & 81.856 & 20.155 & 1.719 & -1.172 \\
\hline 10 & 2.838 & -58.374 & 106.688 & -46.283 & 34.813 & 79.110 & 96.695 & -1.726 & 35.387 & -7.823 & 1.287 & -0.957 \\
\hline 11 & & -75.305 & 104.878 & -44.601 & 30.015 & & 72.364 & 3.526 & & 5.274 & 1.287 & -0.935 \\
\hline 12 & 19.132 & -75.394 & 89.362 & -37.272 & 21.827 & 25.036 & 56.429 & 11.678 & 81.769 & 23.393 & 1.286 & -0.832 \\
\hline 13 & 70.228 & -225.024 & 215.870 & -71.523 & 13.649 & 51.625 & 48.578 & 1.195 & 7.479 & 10.334 & 0.955 & -0.578 \\
\hline
\end{tabular}



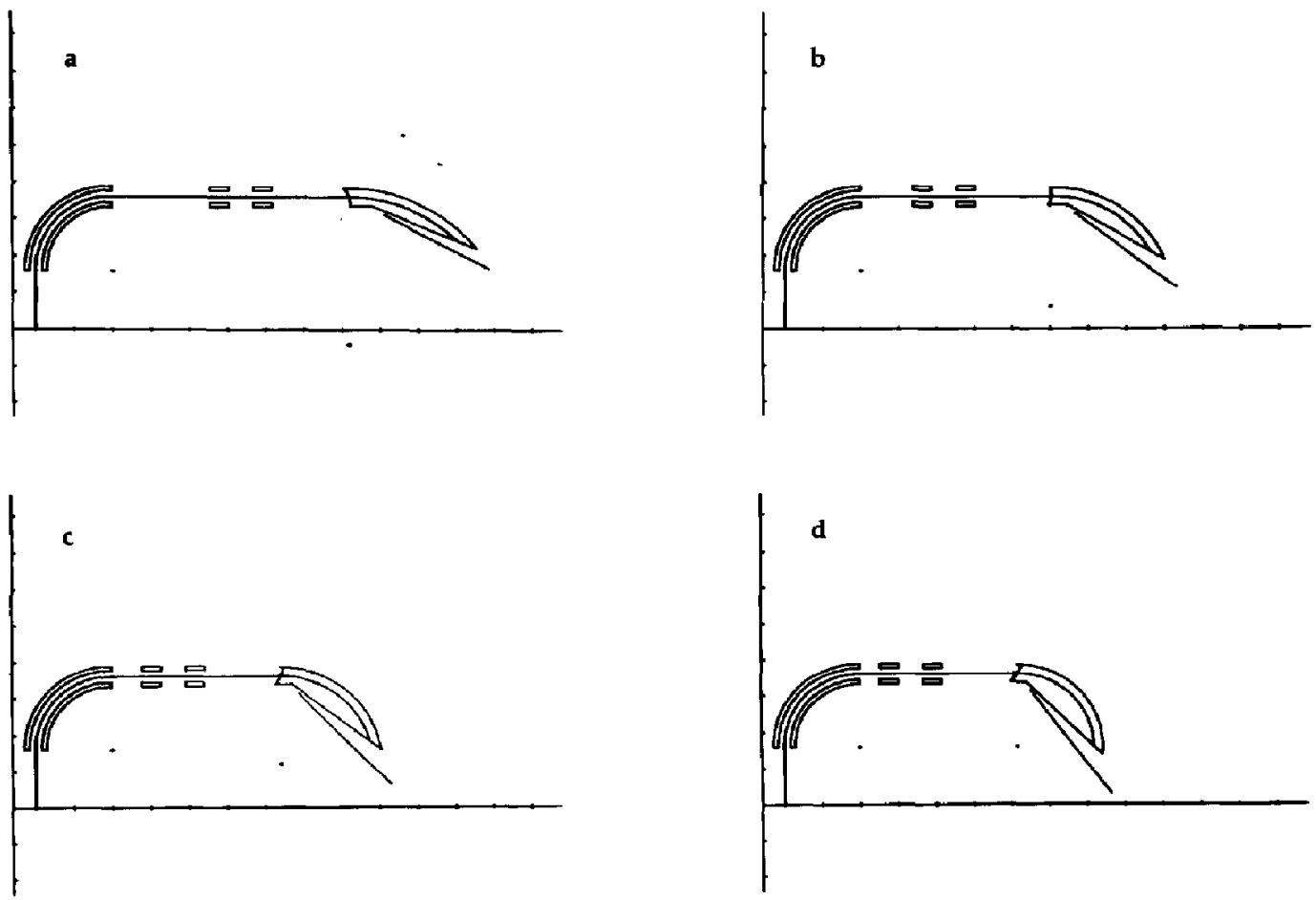

Figure 14. Ion optical block diagram and focal plane of four mass spectrographs: (a) $r_{\mathrm{e}}=1.0$, $\phi_{\mathrm{t}}=90^{\circ}, r_{\mathrm{m}}=2.0, \phi_{\mathrm{m}}=45^{\circ}$; (b) $r_{\mathrm{e}}=1.0, \phi_{\mathrm{e}}=90^{\circ}, r_{\mathrm{m}}=1.5, \phi_{\mathrm{m}}=60^{\circ} ;$ (c) $r_{\mathrm{e}}=1.0, \phi_{\mathrm{e}}=90^{\circ}$, $r_{\mathrm{m}}=1.2, \phi_{\mathrm{m}}=75^{\circ} ;$ (d) $r_{\mathrm{e}}=1.0, \phi_{\mathrm{e}}=90^{\circ}, r_{\mathrm{m}}=1.0, \phi_{\mathrm{m}}=90^{\circ}$.

(overall transfer matrix elements). The ion optical block diagram of four groups are shown in Figure 14. To estimate the actual focusing characteristics of each system, the theoretical mass resolutions for given ion source conditions are given in Table 5 and illustrated in Figure 15. Of the 13 cases, the three important cases are now discussed separately. The particular radius in a magnet shown in Table 1 was first selected in the process of calculation, and the overall geometry was fixed. Then ion optical characters with different radii were calculated under this configuration.

$\phi_{m}=75^{\circ}$ System. We defined the lowest and highest masses to be detected under a constant magnetic field as $0.16 m_{0}$ and $1.14 m_{0}$, respectively, and the overall transfer matrix elements and theoretical mass resolutions are given in Tables 4-6 (seventh, eighth, and ninth lines). The TRIO output $\left(\phi_{\mathrm{c}}=90^{\circ}, \phi_{\mathrm{m}}=75^{\circ}\right)$ is shown in Figure 16. Ion trajectories through this system can be drawn by the newly developed ray-tracing program ELECTRA [12] and is shown in Figure 17. Focusing situations (direction, energy, and double focusing) on the focal plane area can be clearly understood from Figure 17. The beam envelope in the vertical direction is illustrated in Figure 18 in which the vertical focusing character can be seen. The linearity of the focal plane can be estimated in two ways: (1) The focal line is assumed to be a straight line $(y=p x+q)$,

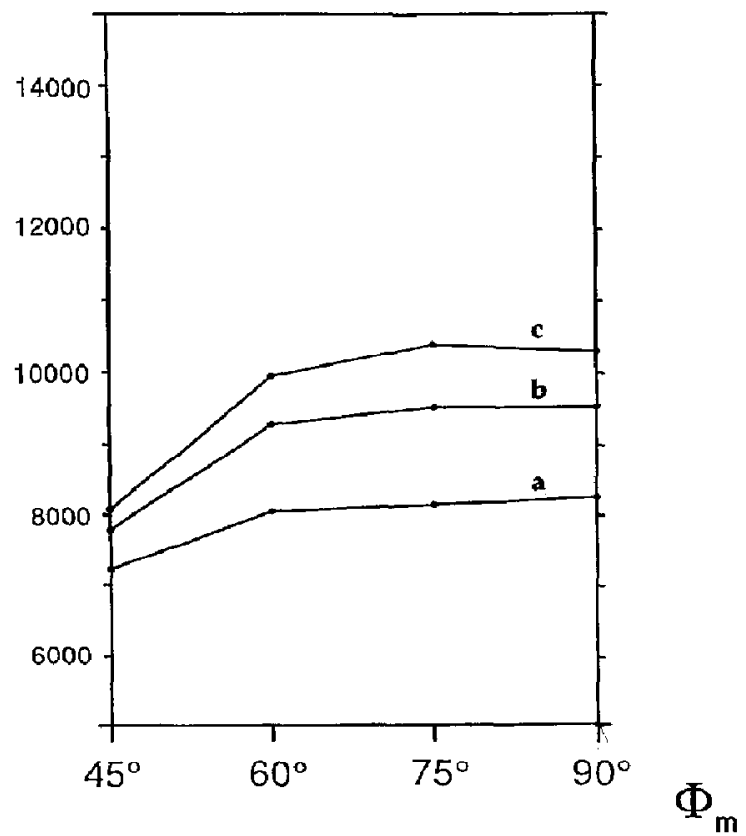

Figure 15. Simulated theoretical mass resolution as the function of the magnetic deflection angle $\phi_{\mathrm{m}}$, where $x_{0}=0.0001 ; \alpha_{0}=$ $0.002 ; \delta=0.002 ; y_{0}=0.001 ; \beta_{0}=0.002:$ (a) $r_{\mathrm{c}}=1.0, \phi_{\mathrm{e}}=60^{\circ}$, $L_{1}=1.14 ;$ (b) $r_{\mathrm{e}}=1.0, \phi_{\mathrm{e}}=75^{\circ}, L_{1}=0.908$; (c) $r_{\mathrm{e}}=1.0, \phi_{\mathrm{e}}=90^{\circ}$, $L_{1}=0.80$. 
DRIFT SPACE

FRINGING FIELD ELECTRIC SECTOR ENTRANCE

CYINDRICAL ELECTRIC SECTOR MAIN

FRINOINO FIELD ELECTFIC SECTOR EXIT

DRIFT SPACE

DRIFT SPACE

FRINUINO FIELD ELECTRIC OUAD ENTRANCE

MAIN ELECTRIC OUAD

FRINOINO FIELD ELECTRIC QUAD EXIT

DRIFT SPACE

FRINOINO FIELD ELECTRIC QUAO ENTRANCE
MAIN ELECTRIC OUAD

FRINGINO FIELD ELECTRIC QUAD EXIT

DRIFT SPACE

FRINOING FIELD MAONETIC SEGTOR ENTRANCE

HOMOGENEOUS HAONETIC SECTOR MAIN FRINOINO FIELD MAGNETIC SECTOR EXIT DRIFT SPACE

A-MATRIX

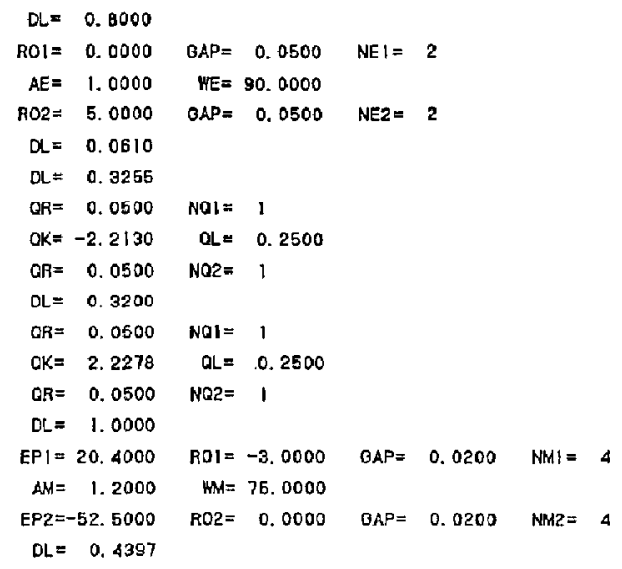

\begin{tabular}{|c|c|c|c|c|c|c|c|c|c|c|}
\hline & $x$ & A & $\mathrm{c}$ & $D$ & $x x$ & $X A$ & $x c$ & $x$ & AA & $A C$ \\
\hline$x$ & 0. 34885 & 0.00000 & 0. 44363 & 0. 00000 & -2.84219 & -3.46165 & -1.85496 & 1. 68328 & -0.05027 & -2.29551 \\
\hline \multirow[t]{2}{*}{ A } & 2. 53392 & 2. 86848 & -0.00064 & -1.54308 & 12. 67247 & 21.61357 & -1.50726 & $-15,6634 \theta$ & 13.87938 & -1.42972 \\
\hline & $A D$ & $\mathrm{CG}$ & $\mathrm{CD}$ & od & $Y Y$ & YB & BB & $\infty x$ & $\infty A$ & $x \times c$ \\
\hline $\mathrm{x}$ & 0.36396 & -0.11085 & 1. 23556 & 0.11337 & -1.35003 & -0.59354 & 0.99845 & 94. 76370 & 245.82537 & -10.81414 \\
\hline \multirow[t]{2}{*}{ A } & -11.21887 & 0.00041 & 1.07904 & 6. 17370 & -2.17471 & -20.04650 & -28.40392 & -211.05463 & -420.90567 & -9.98736 \\
\hline & $\mathrm{XxO}$ & XAA & $X A C$ & XAD & $x 00$ & $\mathrm{xco}$ & XDD & $X Y Y$ & XYB & 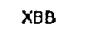 \\
\hline$x$ & -217.12551 & 205. 53684 & -18.62886 & -370.19846 & 1.67169 & 12. 91450 & 176.31252 & 9. 52916 & $-21,8698 i$ & 31.03451 \\
\hline \multirow[t]{2}{*}{$A$} & 557. 74896 & -264.72321 & -17.76880 & 741,26868 & 1. 13142 & 13.90070 & -518.35634 & 8. 11069 & 61.70426 & -47.60922 \\
\hline & AAB & AAC & $A A D$ & $\mathrm{ACO}$ & $A C D$ & $A O D$ & AYY & AYB & $\mathrm{ABB}$ & $\operatorname{ccc}$ \\
\hline$x$ & 68. 68566 & -11.43077 & -157.01005 & 1. 71938 & 0. 45118 & 145.18291 & 27. 27224 & 21.77153 & 30.63828 & 0.05580 \\
\hline \multirow[t]{2}{*}{ A. } & -39.02576 & -10.67352 & 252. 19312 & 1. 07321 & 11.26640 & -338.78122 & -28.04484 & $-121,80364$ & $-243,82752$ & -0.00033 \\
\hline & $\mathrm{CCD}$ & $C D D$ & $\mathrm{CrY}$ & Cri & $\mathrm{CEB}$ & 000 & DYY & DYB & DBE & \\
\hline$x$ & -1.17253 & -4.13577 & 1.60777 & 15.52904 & 22.87647 & -48.25441 & 13. 94642 & 81. 85386 & 20. 14367 & \\
\hline \multirow[t]{2}{*}{ A } & -0.81002 & -5.26692 & 2. 63276 & 10.56231 & 14. B9103 & 151.71276 & -37.00044 & -192.20589 & -127.39446 & \\
\hline & $Y$ & B & $Y X$ & YA & $\mathrm{YC}$ & Yo & $\mathrm{BX}$ & BA & BC & $B D$ \\
\hline$r$ & -2.34400 & 0.00033 & -11.85628 & 5. 33951 & 0.64354 & 24.27150 & -59.29491 & -14.37819 & -0.01594 & 78.11284 \\
\hline a & $-2,22290$ & -0.42629 & -12.39525 & 2. 71836 & 0.03092 & 22,97213 & -54.11029 & $-|4.6052|$ & $-0,13215$ & 69.65016 \\
\hline
\end{tabular}

Figure 16. Output of TRIO for system No. 9 in Tables 4-6: $X, A, C, D, X X, X A, \ldots$, represent the overall transfer matrix elements $T_{x}, T_{x}, T_{y}, T_{\delta}, T_{x x}, T_{x a}, \ldots$, respectively.

and the coefficients $p$ and $q$ can be derived by leastsquare fitting with standard deviations; (2) the focal line is polynomially fitted, and the second-order coefficient is calculated from a fitting routine. The results of these numbers are given in Table 7 . The simulated mass resolution for a wide mass range $\left(r_{\mathrm{m}}=0.4 r_{0}\right.$ $0.6 r_{0}, 0.8 r_{0}, 1.0 r_{0}, 1.2 r_{0}$ ) are represented graphically in Figure 19. In terms of both high mass range and high mass resolution, this is the highest performing configuration. The decrease in mass resolution at lower mass range due to larger aberrations is a problem that has yet to be solved.

$\phi_{m}=45^{\circ}$ System. If heavier ions are required to be analyzed, a larger magnetic radius is needed; but to keep the size of the magnet sensible, a smaller deflection angle must be used. Because third-order image aberrations increase greatly with a smaller magnetic deflection angle, the theoretical mass resolution decreases as shown in Figure 15. By introducing an octapole lens, third-order aberrations can be eliminated considerably at a certain mass range.

$\phi_{m}=90^{\circ}$ System. The original Mattauch-Herzog geometry used a $90^{\circ}$ magnetic sector, and with $s^{\prime}=$ 0 , the focal plane coincided with the magnetic field boundary. This characteristic was ideal for photographic plate detection because this arrangement is free from the fringing field influences. Ion optical calculations were done without the magnetic fringing ficld for both the original and new systems with $\varepsilon^{\prime}=0$ [Tables 1-3 (fifth line) Tables 4-6 (thirteenth line)]. Even in situations where the focal plane is required to coincide with the magnetic boundary, there are consid- 

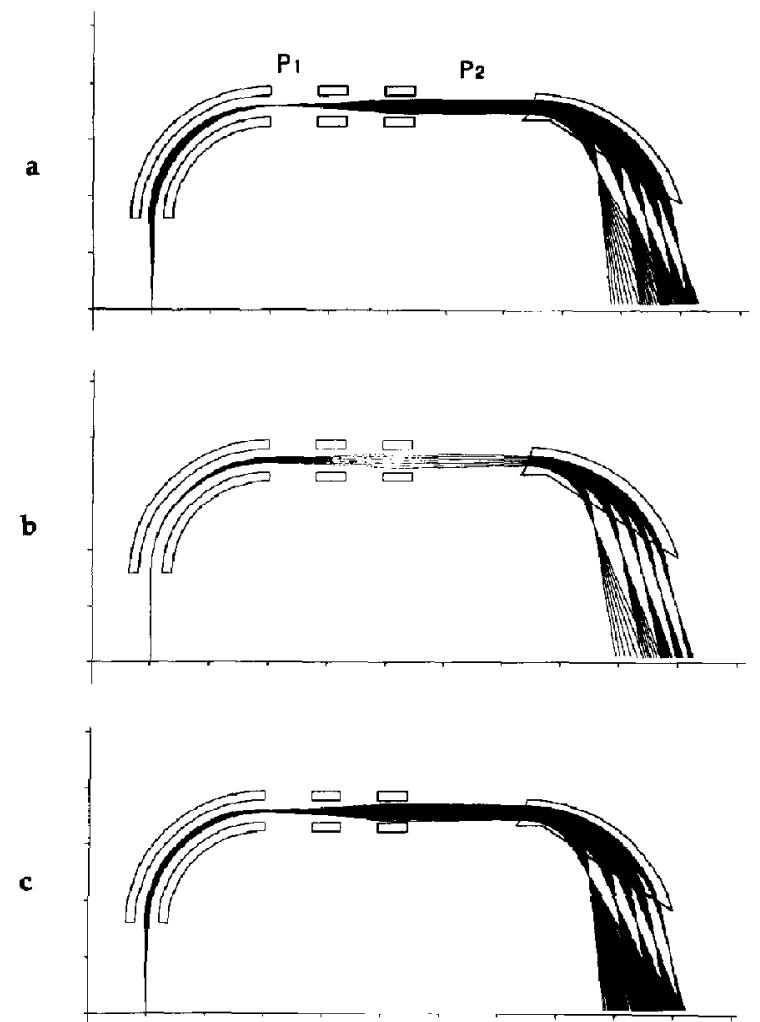

Figure 17. Ion trajectories of ions of different mass $\left(0.16 \mathrm{~m}_{0}\right.$ $\left.0.36 m_{0}, 0.64 m_{0}, m_{0}, 1.14 m_{0}\right)$ and (a) angle, (b) energy, and (c) angle and energy, respectively. A mass spectrograph consists of an electrostatic analyzer $\left(r_{e}=1.0, \phi_{e}=90^{\circ}\right)$, two electric quadrupole lenses, and a homogeneous magnet $\left(r_{\mathrm{m}}=1.2, \phi_{\mathrm{m}}=\right.$ $75^{\circ}$ ). Ion optical parameters and characteristics are shown in Figure 16. Ion beams are focused at the position $P_{1}$ and are parallel at $P_{2}$ in a.

erable improvements in these new designs in terms of mass resolution, ion transmission, and second-order aberrations. The theoretical mass resolutions for the mass range of $0.4 r_{0}$ and $1.2 r_{0}$ of both the original and the new Mattauch-Herzog systems are compared and shown in Figure 19. An ion optical representation of this configuration given in Tables 4-6 (twelfth line) is shown in Figure 14d. The overall transfer matrix elements for different $\phi_{\mathrm{e}}$ are presented in Tables 4-6 (tenth to twelfth lines). It should be noted that the angle of separation $\omega_{i}$ will become large if a larger $\varepsilon^{\prime}$ is given; however, mass resolution cannot be improved

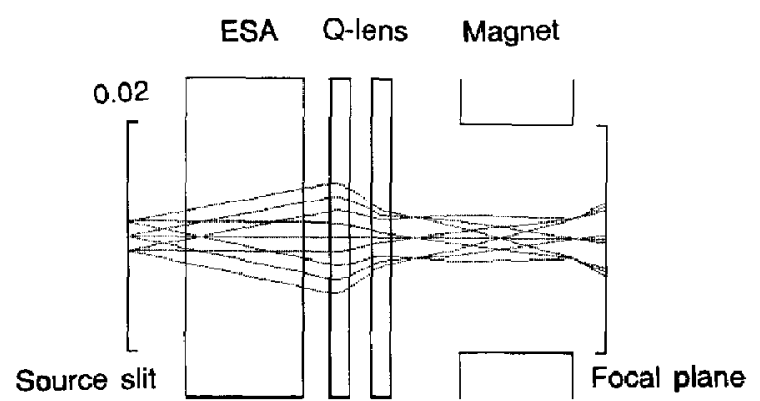

Figure 18. Ion beam envelope in the vertical direction of a system shown in Figure 16.

because of larger aberrations caused by the fringing field influence (Tables $4-6$, twelfth and thirteenth lines).

\section{Discussion and Conclusions}

It is important to classify instruments into several groups to understand the relevant ion optical characteristics, and there are two classes that are pertinent in this case:

1. Normal or inverse combination of ESA and magnet. To satisfy the double-focusing condition, the number of image points between the first and second sectors must be taken into consideration. An "odd" number of images requires a "normal" combination, and an "even" number (including zero) requires inverse geometry. Virtual images should not be counted.

2. Parallel or nonparallel ion beam before the magnet. The double-focusing conditions are classified according to two cases (eqs 8 and 9 or eqs 14 and $15)$, depending on whether the ion beams are parallel or nonparallel before the magnetic field.

It should be noted that the classification is dependent on the ion optical parameters that are most important. For example, in the $\phi_{\mathrm{m}}=75^{\circ}$ system, as shown in Figure 17a, there is an image formed at point $P_{1}$, whereas the ion beam is parallel at point $\boldsymbol{P}_{2}$. 'I'hen, normal deflection must be adopted, and the exit angle $\varepsilon^{\prime \prime}$ is equal to $\phi_{\mathrm{m}} / 2-\pi / 2$; the double-focusing line is then straight regardless of the number of quadrupole lenses. The above two classifications will give us relevant hints when new ion optical designs must be considered.

Table 7. Evaluation of the linearity of focal line for system No. 9 in Tables $4-6^{*}$

\begin{tabular}{lcccc}
\hline & $p$ & $q$ & $\begin{array}{c}\text { Standard } \\
\text { deviation }\end{array}$ & $\begin{array}{c}\text { Second-order } \\
\text { coefficient }\end{array}$ \\
\hline Direction focal line & 0.96572 & 0.00380 & 0.00071478 & 0.000245 \\
Energy focal line & 0.96661 & 0.00263 & 0.00083724 & 0.000474 \\
\hline
\end{tabular}

${ }^{a}$ Calculations were performed with double precision. 


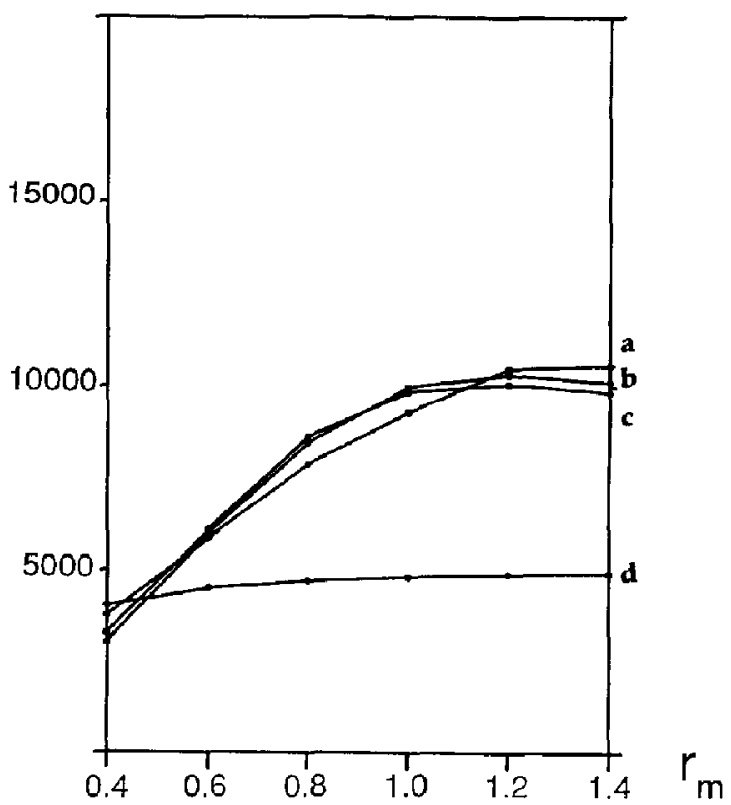

Figure 19. Simulated theoretical mass resolution for different masses $\left(0.16 m_{0}, 0.36 m_{0}, 0.64 m_{0}, m_{0}, 1.14 m_{0}\right)$ under the same source slit condition of $x_{0}=0.0001 ; \alpha_{0}=0.002 ; \delta_{0}=0.002 ; y_{0}=$ 0.001; $\beta_{0}=0.002$ : (a) proposed system No. 9 in Tables 4 6; (b) system No. 13 in Tables 4-6; (c) system No. 12 in Tables 4-6; and (d) the original Mattauch-Herzog system No. 5 in Table 1.

The systems discussed here are planned for use under constant-energy ion beams. Although the important application of the mass spectrograph is as a detector of collision-induced dissociation (CID) ions in MS/MS operation, where constant velocity, wideenergy-spread beam must be analyzed, the present systems discussed here can be used for this purpose; however, CID ions of wide mass range can be detected by using low-energy collision and postacceleration methods because fragment ions can attain almost equal energy.

In summary:

1. Equation 13, which may be used to determine the magnetic field boundary and which satisfies the double-focusing condition for a wide mass range, is derived. The relationship is based on transfer matrix elements and is applicable to any system. One example of Nier-Johnson geometry is illustrated in Figure 6. Although the focal plane is usually curved in these instances, it will be applicable if flexible positionsensitive detectors are developed.

2. A new single-focusing mass spectrograph is proposed that has an intermediate image at the magnet boundary. The direction focal plane is straight and located further from magnetic boundary than the system in which ion beams are parallel before the magnet.

3. New mass spectrographs of a normal combination are proposed that have a straight double-focusing focal line away from the magnet boundary. The ion beam is parallel before the magnet, and the exit angle from the magnet $\varepsilon^{\prime \prime}$ should be equal to $\phi_{\mathrm{m}} / 2-\pi / 2$. These two characteristics are essential to realize a wide-range straight double-focusing line, and 13 examples are shown. Ion optical characteristics (image magnification, vertical focusing, and higher order aberrations) are greatly improved compared with the Mattauch-Herzog system using the same size magnet. A decrease in mass resolution at lower mass ranges has to be solved in future.

4. It became clear that any ESA of different $\phi_{\mathrm{e}}$ will produce almost similar ion optical characteristics if the image magnification $T_{x}$ is identical. This point is helpful when systems have to be designed under restrictions, such as total path length.

5. We improved the original TRIO to TRIODRAWING, which has the capability of graphic display of the calculated results. For example, Figures 6, $7,11,14,17$, and 18 are drawn using TRIO-DRAWING. Visual expression of the results enables straightforward evaluation of each ion optical system. The raytracing code ELECTRA [12] has also been developed. Both programs will be published elsewhere.

\section{Acknowledgments}

We express sincere thanks for valuable discussions to Dr. Toru Sakurai, Osaka University, and Dr. Mike Morris, National Research Council Canada.

\section{References}

1. Hintenberger, H.; König, L. A. Z. Naturforsch. 1957, 12a, $140-147$.

2. Johnson, E. G.; Nier, A. O. Phys. Rev. 1953, 91, 10-17.

3. Mattauch, J.; Herzog, R. Z. Phys. 1934, 89, 786-795.

4. Matsuo, T.; Matsuda, H.; Fujita, Y.; Wollnik, H. Mass Spectrosc. Jpn. 1976, 24, 19-62.

5. Kowalski, S.; Engle, H. A. Nucl. Instrum, Methods 1987, A258, 407 .

6. Wachsmuth, H. W.; Edwald, H. Z. Naturfortch. 1963, 18a, 389-397.

7. Matsuda, H. Mass Spectrosc. Jpn. 1989, 37, 141-148.

8. Hintenberger, H.; König, L. A. Adv. Mass Spectrom. 1959, 1, $16-35$.

9. Takeshita, I. Z. Naturforsch. 1966, 21a, 14-25.

10. Slodzian, G.; Daigne, B.; Girad, F; Bourt, F. Proceedings of the 6ih Internulional Conference on Secondary Ion Mass Spectrametry (SIMS VI); 1987; pp 189-192.

11. Matsuo, T. Mass Spectrosc. Jpn. 1984, 32, 319-326.

12. Ishihara, M.; Matsuo, T. Nucl. Instrum. Methods 1992. B70, $445-450$. 